\title{
RICCI CURVATURE BOUNDS AND EINSTEIN METRICS ON COMPACT MANIFOLDS
}

\author{
MICHAEL T. ANDERSON
}

\section{INTRODUCTION}

Let $M_{0}$ and $M_{1}$ be compact Riemannian manifolds. In [20], Gromov introduced the notion of Lipschitz distance between $M_{0}$ and $M_{1}$, defined by

$$
d_{L}\left(M_{0}, M_{1}\right)=\inf _{f}\left[|\log \operatorname{dil} f|+\left|\log \operatorname{dil} f^{-1}\right|\right],
$$

where $f: M_{0} \rightarrow M_{1}$ is a homeomorphism and $\operatorname{dil} f$ is the dilatation of $f$ given by $\operatorname{dil} f=\sup _{x_{1} \neq x_{2}} \operatorname{dist}\left(f\left(x_{1}\right), f\left(x_{2}\right)\right) / \operatorname{dist}\left(x_{1}, x_{2}\right)$. If $M_{0}$ and $M_{1}$ are not homeomorphic, define $d_{L}\left(M_{0}, M_{1}\right)=+\infty$. Gromov [20] proves the remarkable result that the space of compact Riemannian manifolds $\mathscr{M}(\Lambda, \delta, D)$ of sectional curvature $|K| \leq \Lambda$, injectivity radius $i_{M} \geq \delta>0$, and diameter $d_{M} \leq D$, is $C^{1,1}$ compact with respect to the Lipschitz topology. By $C^{1,1}$ compact we mean that any sequence in $\mathscr{M}(\Lambda, \delta, D)$ has a subsequence which converges, in the Lipschitz topology, to a $C^{\infty}$ manifold $M$ with $C^{0}$ Riemannian metric and $C^{1,1}$ distance function $\rho: M \times M \rightarrow \mathbf{R}$. Related but different proofs of this result obtaining a limit $C^{1, \alpha}, \alpha<1$, Riemannian metric on $M$ appear in [19, 25]. A number of applications of the Gromov compactness theorem have now been obtained, for example in [4, 25]. For an interesting discussion of this result in the context of more general studies, we refer to [30].

An important antecedent of Gromov's compactness theorem is Cheeger's finiteness theorem [8] that the set $\mathscr{M}(\Lambda, v, D)$ of compact Riemannian manifolds of curvature $|K| \leq \Lambda$, volume $V_{M} \geq v$, and diameter $d_{M} \leq D$, has only finitely many diffeomorphism types (cf. also [31]). A basic step in this theorem is a lower bound estimate for the injectivity radius $i_{M} \geq c\left(|K|, d_{M}, V_{M}^{-1}\right)$. In particular, Gromov's compactness theorem may be strengthened to the statement that $\mathscr{M}(\Lambda, v, D)$ is $C^{1,1}$ compact in the Lipschitz topology.

In this paper, we study the question of Lipschitz convergence of compact Riemannian manifolds with bounds imposed on the Ricci curvature Ric in

Received by the editors March 15, 1988 and, in revised form, October 24, 1988.

1980 Mathematics Subject Classification (1985 Revision). Primary 53C25, 58G30, 53C20, $58 \mathrm{D} 17$.

Partially supported by NSF Grant DMS 8701137. 
place of bounds on the sectional curvature. Of course, this is interesting only if $\operatorname{dim} M \geq 4$.

Besides its intrinsic interest, one motivation for this is the study of the existence and the moduli space of Einstein metrics on a compact manifold $M$. Recall that Einstein metrics are exactly critical points of the total scalar curvature functional $\mathscr{R}: \mathscr{M}_{1} / \mathscr{D} \rightarrow \mathbf{R}$,

$$
\mathscr{R}=\int_{M} \tau_{g} d v_{g},
$$

where $\mathscr{M}_{1}$ is the space of Riemannian metrics of volume 1 on $M, \mathscr{D}$ is the diffeomorphism group, acting on $\mathscr{M}_{1}$ by pullback, and $\tau_{g}$ is the scalar curvature. $\mathscr{R}$ may be viewed as a smooth function on $\mathscr{M}_{1}$, and one has [5]

$$
d \mathscr{R}_{g}=\operatorname{Ric}_{g}-\left(\tau_{g} / n\right) g \text {. }
$$

The existence of critical points of a functional can often (but certainly not always) be deduced if the functional satisfies a Morse or Lusternik-Schnirrelman theory. Crucial for this is a condition such as Condition $\mathrm{C}$ of Palais-Smale; namely, in this case, if $g_{i}$ is a sequence of metrics such that $\left|\mathscr{R}\left(g_{i}\right)\right| \leq K$ and $\left\|d \mathscr{R}\left(g_{i}\right)\right\| \rightarrow 0$ as $i \rightarrow \infty$, then a subsequence converges to a critical point of $\mathscr{R}$. Here the norm and topology may be given by a suitable Sobolev or sup norm on $\mathscr{M}_{1}$. This condition then involves bounds on the Ricci tensor, but not on the sectional curvature. Similarly, on the moduli space of Einstein metrics on a given manifold, one has no a priori bounds on the sectional curvature.

Another motivation is the recent result of L. Gao [14] that the space of +1 Einstein metrics on a 4-manifold $M$ is compact in the $C^{\infty}$ topology. This result, the first of its kind on Einstein metrics (known to the author), shows that one can control the geometry of $M$ in terms of the injectivity radius.

Our first result in dimension 4 is as follows. Let $l_{M}$ be the length of the shortest inessential (i.e., null-homotopic) nontrivial geodesic loop on the Riemannian manifold $M$. (If no such loop exists, define $l_{M}$ to be $+\infty$.)

Theorem A. The space of compact 4-dimensional Riemannian manifolds $M$ such that

(i) $\mid$ Ric $\mid \leq c_{1}$,

(ii) $\sup _{r \leq \varepsilon} r^{2(1-4 / q)}\left[\int_{B(r)}\left|D^{2} \mathrm{Ric}\right|^{q / 2}\right]^{2 / q} \leq c_{2}(\varepsilon, q)$, for some $q>4, \varepsilon>0$,

(iii) $l_{M} \geq c_{3}$,

(iv) vol $_{M} \geq c_{4}$,

(v) $\operatorname{diam}_{M} \leq c_{5}$,

(vi) $\operatorname{dim} H_{2}(M, \mathbf{R}) \leq c_{6}$

is $C^{1, \alpha}$ compact in the Lipschitz topology.

Here in (ii), $D^{2}$ Ric is the second covariant derivative of the Ricci tensor. The bounds (i) and (ii) are implied by a bound on the Holder space norm $\|\nabla \mathscr{R}\|_{3, \alpha}$ of the gradient of the total scalar curvature. We note that the bound 
(iii) may be replaced by other bounds. For instance, it may be replaced by $\mathscr{A}_{M}=\inf \left\{\right.$ area $\Sigma:[\Sigma] \neq 0 \in H_{2}(M, \mathbf{Z}) /$ Torsion $\} \geq a>0$, cf. $\S 6$. If $H_{2}(M, \mathbf{Z})$ is torsion, this condition becomes vacuous. There is an analogous result in dimensions $>4$ when the bound on the second Betti number $b_{2}(M)$ is replaced by a bound on the curvature integral $\int_{M}|R|^{n / 2}$, where $R$ is the curvature tensor of $M$.

Theorem $\mathbf{A}^{\prime}$. (I). The space of compact $n$-dimensional Riemannian manifolds $M$ such that

(i) $|\operatorname{Ric}| \leq c_{1}$,

(ii) $\sup _{r \leq \varepsilon} r^{2(1-n / q)}\left[\int_{B(r)}\left|D^{2} \mathrm{Ric}\right|^{q / 2}\right]^{2 / q} \leq c_{2}(\varepsilon, q)$, for some $q>n, \varepsilon>0$,

(iii) $l_{M} \geq c_{3}$,

(iv) vol $_{M} \geq c_{4}$,

(v) $\operatorname{diam}_{M} \leq c_{5}$,

(vi) $\int_{M}|R|^{n / 2} \leq c_{6}$

is $C^{1, \alpha}$ compact in the Lipschitz topology.

(II) If $n$ is odd, condition (iii) on $l_{M}$ may be dropped.

Notice that in Theorem A no assumption is made regarding the sectional curvature, while in Theorem $\mathrm{A}^{\prime}$ a comparatively weak assumption, namely a bound on the scale-invariant integral $\int_{M}|R|^{n / 2} d V$, is made. The quantity in (ii) scales in the same way as the sectional curvature (or the norm of any $(3,1)$ tensor), and again may be replaced by a bound on $\|\nabla \mathscr{R}\|_{3, \alpha}$. Theorems $A$ and $A^{\prime}$ apply naturally to Einstein manifolds, or their products, since then $D$ Ric $\equiv 0$, and lead to the following consequences, proved in $\S 4$.

Corollary B. (1)(a) The space of Einstein metrics of Ricci curvature +1 on compact 4-manifolds $M$ such that $l_{M} \geq c_{1}, \operatorname{vol}_{M} \geq c_{2}$, and $b_{2}(M) \leq c_{3}$ is compact in the $C^{\infty}$ topology. In particular, there are only finitely many diffeomorphism types.

(b) On each component $D$ of the moduli space of +1 Einstein metrics on a given 4-manifold $M$, the function $l_{M}: D \rightarrow \mathbf{R}^{+}$is a proper exhaustion function.

(2) The space of Einstein metrics of Ricci curvature 0 or -1 on compact 4-manifolds $M$ such that $l_{M} \geq c_{1}$, $\operatorname{vol}_{M} \geq c_{2}, \operatorname{diam}_{M} \leq c_{3}$, and $b_{2}(M) \leq c_{4}$ is compact in the $C^{\infty}$ topology. In this case, the function $l_{M}^{-1}+\operatorname{diam}_{M}$ is $a$ proper exhaustion function on each component $D$ of the moduli space.

These results hold with $\mathscr{A}_{M}$ in place of $l_{M}$.

(3) Results (1) and (2) above hold for the space of Einstein metrics on compact $n$-dimensional manifolds $M^{n}$, provided the bound on $b_{2}(M)$ is replaced by a bound on $\int_{M}|R|^{n / 2}$. If $n$ is odd, the lower bound on $l_{M}$ may be dropped. Thus, for example, the function $\int_{M}|R|^{n / 2}$ is a proper exhaustion function on the moduli space of +1 Einstein metrics on a given manifold $M$ in this case. 
(4) (Almost Einstein metrics) There is a constant

$$
\delta=\delta\left(|\operatorname{Ric}|, \sup _{r \leq \varepsilon} r^{2(1-4 / q)}\left\|D^{2} \operatorname{Ric}\right\|_{B(r), q / 2}, l_{M}, \operatorname{vol}_{M}, \operatorname{diam}_{M}, b_{2}(M)\right)
$$

such that if $M$ is a compact 4-manifold with $\int_{M}|\mathrm{Ric}-\lambda g| \leq \delta$, then $M$ admits an Einstein metric with $\mathrm{Ric}=\lambda g$. The same result holds if $M$ is a compact $n$-manifold with the bound on $b_{2}(M)$ replaced by a bound on $\int_{M}|R|^{n / 2}$ (and 4 replaced by $n$ ). Again, if $n$ is odd, the bound on $l_{M}$ may be dropped.

Before proceeding, we make some remarks on the hypotheses of Theorems $A$ and $A^{\prime}$, namely, whether any bound may be dropped in the presence of the others.

Remarks. (i) It is very possible that the two-sided condition $|\mathrm{Ric}| \leq c_{1}$ can be weakened to a lower bound $\mathrm{Ric} \geq-c_{1}$ especially in the presence of the bound (ii).

(ii) It is not known if the bound (ii) is necessary in Theorems A and $\mathrm{A}^{\prime}$, and it would be very interesting to know if, or to what extent, it can be removed. In spirit, it is similar to an $\alpha$-Hölder bound on the Ricci tensor.

(iii) A condition of the type (iii) is necessary in even dimensions. In fact, Tian and Yau [35] construct a noncompact connected family of Kähler-Einstein metrics with $c_{1}>0$ (and so Ricci curvature +1 ) on a simply connected 4manifold $\left(\mathbf{C P}^{2} \# 8 \overline{\mathbf{C P}^{2}}\right)$. The first Chern class degenerates at the boundary so there are sequences with no smoothly convergent subsequences. In particular, $l_{M} \rightarrow 0$ (and $\mathscr{A}_{M} \rightarrow 0$ ) for elements in this family; cf. also [27] and the discussion in $\S 5$.

(iv) The lower bound on the volume is necessary, since for instance any manifold $M=N \times S^{1}$ collapses with bounded curvature, cf. [30].

(v) Similarly, a diameter bound is also necessary. The space of flat metrics on an $n$-torus with $v_{M} \geq c>0$ is still noncompact.

(vi) In dimension 4 , it is an interesting open question whether the assumption (vi) is necessary. It is possible that it is a consequence of the bounds (i), (iv), (v). In higher dimensions, the bound on the curvature integral is also not known to be necessary and it would again be interesting to replace this by a weaker curvature invariant, cf. $\S 4$.

In $\S 5$, we turn to the question of the compactification of the space of Einstein manifolds. Consider for instance Corollary $B(1)(b)$. One is led to study the behavior of metrics in the moduli space $\mathscr{E}^{+1}$ of +1 Einstein metrics on a 4-manifold $M$ as $l_{M} \rightarrow 0$, say at a point $p \in M$. The methods developed in the proof of Theorems A and $\mathrm{A}^{\prime}$ (cf., in particular, Theorem 3.5) tend to indicate that $M$ develops a singularity near $p$; namely, a neighborhood of $p$ is replaced by a cone on a spherical space form. In fact, the following result holds (conjectured independently by $\mathrm{H}$. Nakajima). 
Theorem C. Let $\left\{\left(M_{i}, g_{i}\right)\right\}$ be a sequence of compact connected $n$-dimensional Einstein manifolds, normalized so that $\operatorname{tr} \mathrm{Ric} \in\{-1,0,+1\}$ such that

(i) $\operatorname{vol}_{M_{i}} \geq c_{1}$,

(ii) $\operatorname{diam}_{M_{i}} \leq c_{2}$ if $\operatorname{tr} \mathrm{Ric}=-1$ or 0 ,

(iii) $b_{2}(M) \leq c_{3}$ if $n=4, \int_{M_{i}}|R|^{n / 2} \leq c_{3}$ if $n>4$.

Then a subsequence converges, in the Hausdorff topology, to a connected Einstein orbifold $M_{\infty}$ with a finite number of singular points $\left\{p_{i}\right\} \in M_{\infty}$. If $G_{\infty} \equiv M_{\infty}-\bigcup\left\{p_{i}\right\}$, then $G_{\infty}$ has a $C^{\infty}$ Einstein metric $g_{\infty}$ and there are $C^{\infty}$ embeddings $F_{i}: G_{\infty} \rightarrow M_{i}$, for $i$ sufficiently large, such that $\left(F_{i}\right)^{*} g_{i}$ converges, uniformly on compact subsets in the $C^{k, \alpha}$ topology on $G_{\infty}$, to $g_{\infty}$. Each singular point $p_{i}$ has a neighborhood which is homeomorphic to a cone on a spherical space form $C\left(S^{n-1} / \Gamma\right)$. If the metric $g_{\infty}$ is lifted to $B^{n}-\{0\}$ via $\Gamma$, then there is a $\Gamma$-equivariant diffeomorphism $\phi: B^{n}-\{0\} \rightarrow B^{n}-\{0\}$ such that $\phi^{*} g_{\infty}$ extends smoothly over $\{0\}$ to a smooth Einstein metric on $B^{n}$.

Further, if $n$ is odd, there are no singular points and $M_{\infty}$ is an Einstein manifold diffeomorphic to $M_{i}$, for $i$ sufficiently large. In this case, $\left(M_{i}, g_{i}\right)$ (sub)converges smoothly to $\left(M_{\infty}, g_{\infty}\right)$.

Remarks. (1) Condition (i) is automatically satisfied if $M_{i}=M$ and $g_{i}$ are on a connected component of the moduli space of Einstein metrics on $M$.

(2) The number of singular points $\left\{p_{i}\right\}$ and the orders of the local fundamental groups $\left|\Gamma_{i}\right|$ may be bounded above in terms of the bounds (i), (ii), (iii).

(3) Parts of Theorem C have been proved independently by Nakajima [29], cf. also [2] for a sketch of a proof of Theorem C obtained later, but essentially similar to the proof here.

(4) Recent work of Kobayashi-Todorov [27] indicates that Einstein orbifolds do actually arise as Hausdorff limits of Einstein metrics on K3 surfaces; cf. $\S 5$ for further discussion.

In $\S 6$, we study the moduli space of positive Einstein metrics on compact 4-manifolds of low Euler characteristic.

Theorem D. Let $M$ be a compact 4-manifold with Euler characteristic $0<$ $\chi(M)<4$. Then each component of the moduli space $\mathscr{E}^{+}$of positive Einstein metrics on $M$ is compact in the $C^{\infty}$ topology. Further, there are only finitely many components of the space $\mathscr{E}^{+1}$ of +1 Einstein metrics on $M$ with $\mathrm{vol}_{M} \geq$ $c>0$.

This holds for instance for $M=S^{4}$ or $\mathbf{C P}^{2}$. These results bear some resemblance with the compactness theorems of Uhlenbeck for the space of connections [37] and the space of Yang-Mills fields [36] on principle bundles over a compact manifold. In the latter case, this is, of course, not surprising, since Einstein metrics yield Yang-Mills connections on the tangent bundles. However, spaces of Einstein metrics are likely to be more complicated than spaces 
of Yang-Mills fields, since the (base) metrics may degenerate both locally and globally. The results above indicate what happens when the appropriate bounds are imposed, on the space of Einstein manifolds for instance. It remains open, for example, what happens in Theorem $\mathrm{C}$ when the volume of $\left(M_{i}, g_{i}\right) \rightarrow 0$, or $\operatorname{diam}\left(M_{i}, g_{i}\right) \rightarrow \infty$. This behavior may actually occur on a given manifold $M$, as shown by the examples of Wang and Ziller [38].

The origin of this paper owes much to questions and discussions with L. Gao. His result [14] led the author to consider the more general questions here.

The author would also like to thank S. Bando for enlightening him on the existence of the metrics in [7, 13]; this set the framework for the current work. Finally, I also thank H. Nakajima for his correspondence on these topics as well as the Taniguchi Foundation for making these latter contacts possible.

\section{Preliminary RESUlts}

In this section, we will discuss several preliminary results that will be used for the proof of the main theorems. This section may be skipped and referred back to, when necessary.

2.1. First, we unify the discussion of dimensions. Recall the formula of Avez [5] expressing the Euler characteristic $\chi(M)$ of a compact 4-manifold in terms of a curvature integral;

$$
\chi(M)=\frac{1}{8 \pi^{2}} \int_{M}|R|^{2}-4|\mathrm{Ric}|^{2}+\tau^{2},
$$

where $\tau$ is the scalar curvature. Clearly, $\chi(M) \leq 2+b_{2}(M)$ and $\int_{M} \tau^{2} \leq$ $4 \int_{M} \mid$ Ric $\left.\right|^{2}$. Thus, a bound on $\int_{M} \mid$ Ric $\left.\right|^{2}$ and $b_{2}(M)$ implies a bound on $\int_{M}|R|^{2}$. The Bishop comparison theorem (cf. $\S 2.5$ ) implies there is a bound $\operatorname{vol}_{M} \leq c\left(\operatorname{inf~Ric}_{M}, \operatorname{diam}_{M}\right)$, so that the bounds (i), (v), (vi) imply a bound on $\int_{M}|R|^{2}$.

2.2. The hypotheses (i), (iv), ( $v$ ) in Theorems $\mathbf{A}$ and $\mathbf{A}^{\prime}$ lead to a lower bound on the isoperimetric and Sobolev constants of $M$. In fact, let $h_{M}$ be the isoperimetric constant given by

$$
h_{M}=\inf _{S} \frac{[\operatorname{vol} S]^{n}}{\left[\min \left(\operatorname{vol} M_{1}, \operatorname{vol} M_{2}\right)\right]^{n-1}},
$$

where $S$ varies over closed hypersurfaces of $M$ such that $M-S=M_{1} \cup$ $M_{2}$. Croke [10, Theorem 13] shows that $h_{M}$ is bounded below by a constant depending only on a lower bound for the Ricci curvature and volume, and an upper bound on the diameter. In particular, if $B_{x}(r)$ is a geodesic ball of radius $r$ about $x \in M$ and $S_{x}(r)=\partial B_{x}(r), v(r)=\operatorname{vol} B_{x}(r)$, then it follows that $\left(v^{\prime}(r)\right)^{n} / v(r)^{n-1} \geq h_{M}$ for $v(r)<\frac{1}{2} \operatorname{vol} M$. Integrating this inequality, one obtains

$$
v(r) / r^{n} \geq c_{M}
$$


if $v(r)<\frac{1}{2} \operatorname{vol} M$. Also, it is well known that a lower bound for $h_{M}$ gives a lower bound for the Sobolev constant $c_{S}$ of $M$. In fact, cf. [40],

$$
\|f\|_{2 n /(n-2)} \leq \frac{1}{c_{S}}\|d f\|_{2}+\operatorname{vol}_{M}^{-2 / n}\|f\|_{2},
$$

for any Lipschitz function for $M$. Note that the bounds in (2.3) and (2.4) are scale invariant.

2.3. A basic tool in the arguments to follow will be the equation for the (rough) Laplacian of the curvature tensor $R$ of $M$. It is shown in [21, Lemma 7.2] that

$$
\Delta R=R * R+R * \mathrm{Ric}+P^{2}(\mathrm{Ric}),
$$

where $A * B$ denotes a linear combination of tensors $A, B$ obtained by contracting $A, B$ with the metric $g$ and $P^{2}$ (Ric) is a linear combination of second covariant derivatives of the Ricci tensor. In particular, one obtains

$$
|\Delta R| \leq c_{1}\left|D^{2} \mathrm{Ric}\right|+c_{2}|R|^{2},
$$

where $c_{1}$ and $c_{2}$ are constants depending only on dimension. One has $\langle\Delta R, R\rangle$ $+|D R|^{2}=\frac{1}{2} \Delta|R|^{2}=|R| \Delta|R|+|d| R||^{2}$. An application of the Schwartz inequality shows $\left.|d| R\right|^{2} \leq|D R|^{2}$ so that from (2.5) one has

$$
\Delta|R|+c_{1}\left|D^{2} \mathrm{Ric}\right|+c_{2}|R|^{2} \geq 0 .
$$

Elliptic inequalities of this type have now been used in many geometric contexts to derive pointwise bounds, the basic idea going back to Uhlenbeck [32]. Since such a pointwise bound is crucial in our arguments, we will include a full proof, following the lines of [16, Theorem 8.17].

Lemma 2.1. There is a constant $C=C\left(n, c_{S}\right)$ and $\varepsilon_{0}=\varepsilon_{0}\left(n, c_{S}\right)$ such that if $B(t)$ is a geodesic ball of radius $t$ in $M$ and

$$
\int_{B(t)}|R|^{n / 2} d V<\varepsilon_{0}
$$

then

$$
\sup _{B(t / 2)}|R|^{2} \leq C \cdot\left[\frac{1}{t^{2}} \int_{B(t)}|R|^{n / 2}+t^{2 \delta}\left[\int_{B(t)}\left|D^{2} \mathrm{Ric}\right|^{q / 2}\right]^{q / 2}\right]
$$

for a fixed $q>n$ and $\delta=1-\frac{n}{q}$.

Proof. It simplifies matters if we assume $t=1$. Note that inequality (2.6), condition (2.7) and claim (2.8) are all scale invariant, so that by rescaling the metric on $M$, we may assume that $t=1$.

Let $k=\left(\int_{B(1)}\left|D^{2} \mathrm{Ric}\right|^{q / 2}\right)^{2 / q}$ and let $u=|R|+k$. First multiply (2.6) by $\zeta^{2} u^{\alpha}, \alpha \geq 1$, where $\zeta$ is a cutoff function of compact support in $B(1)$, to be determined below. Integrating by parts, one obtains

$$
\frac{4 \alpha}{(\alpha+1)^{2}} \int \zeta^{2}\left|d u^{(\alpha+1) / 2}\right|^{2}-2 \zeta u^{\alpha}|d \zeta||d u| \leq c_{3} \int \zeta^{2} u^{\alpha}\left[\left|D^{2} \operatorname{Ric}\right|+|R|^{2}\right]
$$


By the Young inequality

$$
2 \zeta u^{\alpha}|d \zeta||d u| \leq 2\left(u^{(\alpha+1) / 2}|d \zeta|\right)^{2}+\frac{2 \zeta^{2}}{(\alpha+1)^{2}}\left|d u^{(\alpha+1) / 2}\right|^{2}
$$

so that one obtains

$$
\frac{4\left(\alpha-\frac{1}{2}\right)}{(\alpha+1)^{2}} \int \zeta^{2}\left|d u^{(\alpha+1) / 2}\right|^{2} \leq c_{3} \int \zeta^{2} u^{\alpha}\left[\left|D^{2} \operatorname{Ric}\right|+|R|^{2}\right]+2 u^{\alpha+1}|d \zeta|^{2} .
$$

This gives the estimate

$$
\int\left|d \zeta u^{(\alpha+1) / 2}\right|^{2} \leq c_{4} \cdot \alpha \int \zeta^{2} u^{\alpha}\left[\left|D^{2} \operatorname{Ric}\right|+|R|^{2}\right]+u^{\alpha+1}|d \zeta|^{2},
$$

so that by the Sobolev inequality $(2.4)$

$$
\begin{aligned}
& {\left[\int\left(\zeta u^{(\alpha+1) / 2}\right)^{2 n /(n-2)}\right]^{(n-2) / n}} \\
& \quad \leq c_{5} \alpha \int \zeta^{2} u^{\alpha}\left[\left|D^{2} \operatorname{Ric}\right|+|R|^{2}\right]+u^{\alpha+1}\left[|d \zeta|^{2}+\zeta^{2}\right] .
\end{aligned}
$$

First we set $\alpha+1=n / 2$. Then

$$
\begin{aligned}
\int \zeta^{2}|R|^{2} u^{\alpha} & \leq \int \xi|R| \zeta^{2} u^{\alpha+1} \\
& \leq\left(\int\left(\xi|R|^{n / 2}\right)^{2 / n}\right) \cdot\left(\int\left(\zeta^{2} u^{\alpha+1}\right)^{n /(n-2)}\right)^{(n-2) / n},
\end{aligned}
$$

where $\xi$ is an auxiliary cutoff function with $\xi \equiv 1$ on supp $\zeta$. Since $\int(\xi|R|)^{n / 2}$ $\leq \varepsilon_{0}$, if $\varepsilon_{0}<\frac{1}{2} c_{5}\left(\frac{n}{2}-1\right)$, this term may be absorbed into the left side of (2.9). Next,

$$
\begin{aligned}
\int\left(\zeta^{2}\left|D^{2} \operatorname{Ric}\right|\right) u^{(n-2) / 2} & \leq\left(\int\left|D^{2} \operatorname{Ric}\right|^{n / 2}\right)^{2 / n} \cdot\left(\int \zeta^{2} u^{n / 2}\right)^{(n-2) / n} \\
& \leq \operatorname{vol} B(1)^{1-n / q} k\left[\left\|\zeta^{2}|R|\right\|_{n / 2}+k\right]^{(n-2) / n} .
\end{aligned}
$$

Further, $\int u^{\alpha+1}|d \zeta|^{2} \leq c_{6}\left[\varepsilon_{0}+k\right]$, where $c_{6}$ depends on $|d \zeta|$. If we set $\rho_{0}=$ $(n / 2)(n /(n-2))$, the above estimates combine to give the bound

$$
\|\zeta u\|_{\rho_{0}} \leq c_{7}\left[\varepsilon_{0}+k\right] \text {. }
$$

Now we return to (2.9) with $\alpha+1>\frac{n}{2}$. Note that

$$
u^{\alpha}\left[|R|^{2}+\left|D^{2} \operatorname{Ric}\right|\right] \leq u^{\alpha+1}\left[u+\frac{\left|D^{2} \mathrm{Ric}\right|}{k}\right] \text {, }
$$

so that

$$
\int \zeta^{2} u^{\alpha}\left[|R|^{2}+\left|D^{2} \mathrm{Ric}\right|\right] \leq\left\|\zeta\left(u+\frac{\left|D^{2} \mathrm{Ric}\right|}{k}\right)\right\|_{q / 2} \cdot\left\|\zeta u^{(\alpha+1) / 2}\right\|_{2 q /(q-2)}^{2}
$$


Then

$$
\left\|\zeta\left(u+\frac{\left|D^{2} \mathrm{Ric}\right|}{k}\right)\right\|_{q / 2} \leq\|\zeta u\|_{q / 2}+\left\|\zeta \mid D^{2} \operatorname{Ric}\right\|_{q / 2} \cdot \frac{1}{k} \leq c_{8}\left[\varepsilon_{0}+k\right],
$$

provided $\frac{q}{2} \leq \rho_{0}$. Further, using the interpolation inequality $\|f\|_{s} \leq \varepsilon\|f\|_{r}+$ $\varepsilon^{-\mu}\|f\|_{t}$, where $r>s>t, \mu=\left(\frac{1}{r}-\frac{1}{s}\right) /\left(\frac{1}{s}-\frac{1}{t}\right)$ with $r=2 n /(n-2), s=$ $2 q /(q-2), t=2$, we obtain from (2.9)-(2.11) that

$$
\begin{aligned}
& {\left[\int\left(\zeta u^{(\alpha+1) / 2}\right)^{2 n /(n-2)}\right]^{(n-2) / n} \leq c_{9} \cdot \alpha\left[\left(\varepsilon\left\|\zeta u^{(\alpha+1) / 2}\right\|_{2 n /(n-2)}+\varepsilon^{-\mu}\left\|\zeta u^{(\alpha+1) / 2}\right\|_{2}\right]^{2}\right.} \\
& +\int u^{\alpha+1}\left[|d \zeta|^{2}+\zeta^{2}\right]
\end{aligned}
$$

where $\mu=n /(q-n)$. If we choose $\varepsilon^{2}=\frac{1}{4}\left(c_{9} \alpha\right)^{-1}$, then the first term on the right side of (2.12) may again be absorbed into the left side. We set $v=u^{(\alpha+1) / 2}$ and $\chi=n /(n-2)$ and thus obtain

$$
\|\zeta v\|_{2 \chi} \leq c_{10} \alpha\left(1+\alpha^{\mu}\right)\left[\|(\zeta+|d \zeta|) v\|_{2}\right] .
$$

We may choose the cutoff function $\zeta=\zeta(r)$, where $r$ is the distance to $0 \in$ $B(1)$, and require $\zeta(r) \equiv 1$ for $r \leq r^{-}$with $r^{-} \geq \frac{1}{2}, \zeta(r) \equiv 0$ for $r \geq r^{+}$, where $r^{+} \leq 1$ and $|d \zeta| \leq c /\left(r^{+}-r^{-}\right)$. Let $T(p, r)=\left(\int_{B(r)} u^{p}\right)^{1 / p}$. Then (2.13) gives

$$
T\left(\chi \dot{p}, r^{-}\right) \leq\left(\frac{c_{11} p\left(1+p^{\mu}\right)}{r^{+}-r^{-}}\right)^{2 / p} T\left(p, r^{+}\right) .
$$

This inequality may now be iterated in the standard fashion. Let $p_{1}=\frac{n}{2}$, $p_{m}=\chi^{m} p, r_{m}^{-}=\frac{1}{2}+2^{-(m+3)}, r_{m}^{+}=1-2^{-(m+3)}$. We then obtain

$$
T\left(\chi^{m} \frac{n}{2}, \frac{1}{2}\right) \leq\left(c_{12} \chi\right)^{2\left[1+\sum_{1}^{m} i \chi^{-i}\right]} T\left(\frac{n}{2}, 1\right) \leq c_{13} \cdot T\left(\frac{n}{2}, 1\right) .
$$

Letting $m \rightarrow \infty$ gives

$$
\sup _{B(1 / 2)} u \leq c \cdot\left(\int_{B(1)} u^{n / 2}\right)^{2 / n} .
$$

Lemma 2.1 then follows by applying the triangle inequality to the integral in (2.15).

2.4. We will make frequent use of a local lower bound estimate on the injectivity radius of a manifold, due to Cheeger, Gromov, and Taylor [9, Theorem 4.3]. Namely, let $N$ be a complete manifold and $p \in N$. Let $l_{p}$ denote the length of the shortest geodesic loop in $N$ based at $p$. If $K_{N} \leq \Lambda^{2}$ on $B_{p}(r)$, and $r \leq l_{p} / 2$, then an estimate of Klingenberg [26, Lemma 1], implies that the injectivity radius $i_{p}$ of $N$ at $p$ satisfies

$$
i_{p} \geq \min \left(r, \frac{\pi}{\Lambda}\right) \text {. }
$$


Now suppose $\left|K_{N}\right| \leq \Lambda^{2}$ on $B_{p}(r)$, where $r \leq \frac{\pi}{\Lambda}$. Choose any numbers $r_{0}$ and $s$ such that $r_{0}+2 s \leq r, r_{0} \leq \frac{r}{4}$. Then one has the estimate [9]

$$
l_{p} \geq r_{0}\left[1+v^{-\Lambda}\left(r_{0}+s\right) / v_{p}(s)\right]^{-1},
$$

where $v_{p}(s)$ is the volume of $B_{p}(s)$ and $v^{-\Lambda}\left(r_{0}+s\right)$ is the volume of the geodesic ball of radius $r_{0}+s$ in the space form of constant curvature $-\Lambda$.

In particular, a lower bound on $v_{p}(s)$ and the bound $\left|K_{N}\right| \leq \Lambda^{2}$ imply a lower bound on the injectivity radius of $N$ of $p$. Note that from (2.3), one obtains a lower bound for $v_{p}(s)$ (depending only on $s$ ), from bounds $\operatorname{vol}_{N} \geq c_{4}, \operatorname{diam}_{N} \leq c_{5}\left(\right.$ since $\left.\left|K_{N}\right| \leq \Lambda^{2}\right)$.

2.5. We will also make use of the well-known Bishop comparison theorem [6, 9]. If $M$ is a complete manifold with $\operatorname{Ric}_{M} \geq(n-1) \lambda$, then

$$
\frac{v(r)}{v^{\lambda}(r)} \downarrow
$$

where $v(r), v^{\lambda}(r)$ are as in $\S 2.4$. Clearly $\lim _{r \rightarrow 0} v(r) / v^{\lambda}(r)=\omega_{n}$, the volume of the unit $n$-ball in $\mathbf{R}^{n}$. If $v(r) / v^{\lambda}(r) \equiv \omega_{n}$ for all $r$, then $M$ is isometric to the simply connected space form of constant curvature $\lambda$. We note, in particular, that if $\operatorname{Ric}_{M} \geq 0$, then $v(r) / r^{n} \downarrow$.

2.6. We will need to apply a local form of the Gromov compactness theorem (related to $[20,8.2]$ ) a number of times. First, we give a definition.

Definition. A sequence of Riemannian manifolds $V_{i}$ converges uniformly on compact sets in the $C^{k, \alpha}$ topology to a Riemannian manifold $V$ if, for any compact domain $D \subset V$ and $i$ sufficiently large, there are compact domains $D_{i} \subset V_{i}$ and $C^{k, \alpha}$ diffeomorphisms $F_{i}: D \rightarrow D_{i}$ such that the pull-back $\left(F_{i}\right)^{*} g_{i}$ of the metrics on $D_{i}$ to $D$ converge, in the $C^{k, \alpha}$ topology on $D$, to the metric on $D \subset M$.

Theorem 2.2 (Gromov compactness). Let $V_{i}$ be a sequence of closed $C^{\infty}$ Riemannian manifolds and $\Omega_{i}$ a sequence of domains in $V_{i}$, with smooth boundary $\partial \Omega_{i}$. Suppose, for all $i$,

(i) $\left|D^{l} R\right|(x) \leq \Lambda(l), l=0, \ldots, k$,

(ii) $\operatorname{Inj}(x) \geq c_{1}$,

(iii) $c_{2} \leq \operatorname{vol} \Omega_{i} \leq c_{3}$

for all $x \in \Omega_{i}$, where $D^{l} R$ is the lth covariant derivative of the curvature tensor on $V_{i}$. Then given $\varepsilon>0$, the Riemannian manifolds $\Omega_{i}(\varepsilon) \equiv\{x \in$ $\left.\Omega_{i}: \operatorname{dist}\left(x, \partial \Omega_{i}\right)>\varepsilon\right\}$ (assumed nonempty) have a subsequence which converges, uniformly on compact sets in the $C^{k, \alpha}$ topology, to a $C^{k+1, \alpha}$ Riemannian manifold $\Omega_{\infty}(\varepsilon)$. In particular, $\Omega_{i}(\varepsilon)$ is diffeomorphic to $\Omega_{\infty}(\varepsilon)$ for $i$ sufficiently large. 
Although this specific form of the Gromov compactness theorem does not seem to appear in the literature, a number of proofs that do appear are easily adapted to give a proof of this situation. For completeness, we give a proof of Theorem 2.2, following closely the lines of [24].

First, we recall the following result from [23].

Fact. There are positive constants $\delta_{0}$ and $c_{0}$, depending on the bounds (i), (ii), (iii), and $\varepsilon$, such that for any $\delta \leq \delta_{0}$, there is a harmonic coordinate chart $H=\left(h_{1}, \ldots, h_{n}\right): B_{x}(\delta) \rightarrow \mathbf{R}^{n}$ satisfying

(1) $c_{0}^{-1} \operatorname{dist}(x, y) \leq|H(y)| \leq c_{0} \operatorname{dist}(x, y)$,

(2) $c_{0}^{-1}|\xi|^{2} \leq \sum g_{i j} \xi^{i} \xi^{j} \leq c_{0}|\xi|^{2}$, where $g_{i j}=g\left\langle\nabla h_{i}, \nabla h_{j}\right\rangle$,

(3) $\left\|g_{i j}\right\|_{C^{k+1, \alpha}\left(B_{x}(\delta)\right)} \leq c_{0}$,

(4) if $f$ is any harmonic function in $B_{x}(\delta)$, then

$$
\|f\|_{C^{k+2, \alpha}\left(B_{x}(\delta / 2)\right)} \leq c_{0}|f|_{C^{0}}
$$

for any $x \in \Omega_{i}(\varepsilon)$.

Proof of Theorem 2.2. Let $\delta_{1}=\frac{1}{2} c_{0}^{-5} \delta_{0}$, where $c_{0}, \delta_{0}$ are chosen from the above fact, and we will also assume $\delta_{0}<\varepsilon / 2$. In each domain $\Omega=\Omega_{i}(\varepsilon)$, choose a maximal $\delta_{1} / 2$ separated set $\Gamma$ of points $\left\{x_{j}\right\}$. Thus, dist $_{\Omega}\left(x_{j}, x_{k}\right)>$ $\delta_{1} / 2$ and $\operatorname{dist}_{\Omega}(x, \Gamma)<\delta_{1}$. In particular, the balls $B_{x_{j}}\left(\delta_{1} / 4\right), x_{j} \in \Gamma$, are disjoint and the balls $B_{x_{j}}\left(\delta_{1}\right)$ cover $\Omega_{i}(\varepsilon)$. By the Rauch comparison theorem, $\operatorname{vol} B_{x_{j}}\left(\delta_{1} / 4\right) \geq c(\Lambda) \cdot \delta_{1}^{n}$ and $\operatorname{vol} B_{x_{j}}\left(\delta_{1}\right) \leq c^{\prime}(\Lambda) \cdot \delta_{1}^{n}$. Thus, by (iii), there is a fixed upper bound $Q$ to the cardinality of $\Gamma$ and by passing to a subsequence, we may assume $\# \Gamma=\# \Gamma_{i}(\varepsilon)=Q$ ( $\varepsilon$ fixed). Let $U=U_{i}=\bigcup_{j=1}^{Q} B_{x_{j}}\left(\delta_{1}\right)$, so that $U_{i}$ covers $\Omega_{i}(\varepsilon)$, but is contained in $\Omega_{i}(\varepsilon / 2)$.

For each $x_{j}$, we have harmonic coordinates $H_{j}: B_{j}\left(x_{j}\right) \rightarrow \mathbf{R}^{n}$ satisfying

$$
B^{n}\left(c_{0}^{-1} \delta\right) \subset H_{j}\left(B_{x_{j}}(\delta)\right) \subset B^{n}\left(c_{0} \delta\right)
$$

for $\delta<\delta_{0}$ and $B^{n}(s)$ the ball of radius $s$ about 0 in $\mathbf{R}^{n}$. Let $\zeta$ be a fixed smooth cutoff function satisfying $\zeta(t) \equiv 1$ on $\left[0, \delta_{2}\right], \zeta(t) \equiv 0$ on $\left[\delta_{3}, \infty\right)$, where $\delta_{2}=c_{0}^{3} \delta_{1}, \delta_{3}=c_{0}^{4} \delta_{1}$. Define $\zeta_{j}: U \rightarrow \mathbf{R}$ by $\zeta_{j}(x)=\zeta\left(\left|H_{j}(x)\right|\right)$, so that $\operatorname{supp} \zeta_{i} \subset B_{x_{j}}\left(\delta_{5}\right)$ and $\zeta_{j} \equiv 1$ on $B_{x_{j}}\left(\delta_{4}\right)$, where $\delta_{5}=c_{0}^{5} \delta_{1}, \delta_{4}=c_{0}^{2} \delta_{1}$.

For each $U=U_{i}$, we may define a smooth map $E_{U}: U \rightarrow \mathbf{R}^{N}(N=$ $n Q+Q)$, by

$$
E_{U}(x)=\left(\zeta_{1}(x) \cdot H_{1}(x), \ldots, \zeta_{Q}(x) \cdot H_{Q}(x), \zeta_{1}(x), \ldots, \zeta_{Q}(x)\right) .
$$

It is clear that $E_{U}$ is an embedding of $U$ into a ball $B^{N}\left(r_{0}\right)$ of fixed size in $\mathbf{R}^{N}$. Now observe that for any $x_{j} \in \Gamma, E_{U}\left(B_{x_{j}}\left(\delta_{4}\right)\right)$ is naturally a graph over $H_{j}\left(B_{x_{j}}\left(\delta_{4}\right)\right) \subset \mathbf{R}^{n}=\left(\mathbf{R}^{N}\right)_{j} \subset \mathbf{R}^{N}$. Namely, for $x \in H_{j}\left(B_{x_{j}}\left(\delta_{4}\right)\right)$,

$$
\begin{array}{r}
E_{U}\left(B_{x_{j}}\left(\delta_{4}\right)\right)=\left\{\left(f_{1}(x) F_{1}(x), \ldots, f_{j-1}(x) F_{j-1}(x), x, f_{j+1}(x) F_{j+1}(x),\right.\right. \\
\left.\left.\ldots, f_{Q}(x) F_{Q}(x), f_{1}(x), \ldots, f_{Q}(x)\right)\right\},
\end{array}
$$


where $F_{l}=H_{l} \circ H_{j}^{-1}$ and $f_{l}=\zeta\left(\left|F_{l}\right|\right)$. Further, note that there is a fixed lower bound on the size of $H_{j}\left(B_{x_{j}}\left(\delta_{4}\right)\right)$; in fact, $H_{j}\left(B_{x_{j}}\left(\delta_{1}\right)\right) \subset B^{n}\left(c_{0} \delta_{1}\right) \subset$ $H_{j}\left(B_{x_{j}}\left(\delta_{4}\right)\right)$. Clearly, the balls $B_{x_{j}}\left(\delta_{4}\right)$ cover $U$. Since the maps $H_{j}$ are harmonic, the maps $F_{j}$ and $f_{j}$ have uniformly bounded $C^{k+2, \alpha}$ norms, by (4) of the fact. In particular, all maps $E_{U_{i}}$ have uniformly bounded $C^{k+2, \alpha}$ norm.

We will now identify $U_{i}$ with its image $D_{i}$ in $\mathbf{R}^{N}$ under $E_{U_{i}}$ and the metric $g_{i}$ on $U_{i}$ with its image (call it again $g_{i}$ ), under the push forward $\left(E_{U_{i}}\right)_{*} g_{i}$ on $D_{i}$. We then have a covering of $D_{i}$ by neighborhoods $N_{j}=N_{j}(i)$ which are graphs over a fixed domain $B^{n}\left(\delta_{6}\right) \subset\left(\mathbf{R}^{n}\right)^{j} \subset \mathbf{R}^{N}$, with $\delta_{6}=c_{0} \delta_{1}$, by graphing functions with uniformly bounded $C^{k+2, \alpha}$ norm. We may apply the ArzelaAscoli theorem to each graphing chart and obtain a subsequence of $\left\{D_{i}\right\}$ which converges, in the $C^{k+2, \alpha^{\prime}}$ topology, for $\alpha^{\prime}<\alpha$ to an embedded graph $D_{\infty}$ of class $C^{k+2, \alpha^{\prime}}$. For $i$ sufficiently large, any compact subset of $D_{i}$ may then be graphed over the normal exponential map $X_{\infty}$ to $D_{\infty}$, so that, pushing forward the metric $g_{i}$ to $D_{\infty}$ by $X_{\infty}$, we obtain a sequence of $C^{k+1, \alpha^{\prime}}$ metrics, also called $g_{i}$, on any given compact subset $K \subset D_{\infty}$.

We will now verify that this sequence of metrics has a subsequence, converging uniformly in the $C^{k+1, \alpha}$ topology on $K$, to a $C^{k+1, \alpha}$ metric on $K$. To see this, recall $N=Q n+n$ and let $P^{j}$ be the orthogonal projection of $\mathbf{R}^{N}$ onto $\left(\mathbf{R}^{n}\right)^{j}, 1 \leq j \leq Q$. Let $\Pi_{i}^{j}$ be the restriction of $P^{j}$ to the geodesic ball $B_{x_{j}}\left(\delta_{4}\right) \subset U_{i} \cong D_{i} \subset \mathbf{R}^{N}$. Consider the sequence of metric tensors

$$
h_{\alpha \beta}^{i}=\left(\Pi_{i}^{j}\right)_{*} g_{i}\left(\frac{\partial}{\partial x_{\alpha}}, \frac{\partial}{\partial x_{\beta}}\right)
$$

defined on $B^{n}\left(\delta_{6}\right) \subset\left(\mathbf{R}^{n}\right)^{j}$. To compare these metrics, note that the maps $\phi_{i}^{j}=P^{j} \circ X_{\infty} \circ\left(\Pi_{i}^{j}\right)^{-1}$ are $C^{k+2, \alpha}$ diffeomorphisms from $B^{n}\left(\delta_{6}\right)$ into $\mathbf{R}^{n}$, with uniformly bounded $C^{k+2, \alpha}$ norm in $i$, for each fixed $j$. Thus, they converge to the identity in the $C^{k+2, \alpha^{\prime}}$ topology. It follows that the $C^{k+1, \alpha}$ norms of $h_{\alpha \beta}^{i}$ on $B^{n}\left(\delta_{6}\right)$ are uniformly bounded, so by the Arzela-Ascoli theorem, a subsequence converges to a $C^{k+1, \alpha}$ metric $h_{\alpha, \beta}^{\infty}$ in the $C^{k+1, \alpha^{\prime}}$ topology on $B^{n}\left(\delta_{6}\right)$. Taking then $\left(\Pi_{i}^{j}\right)^{*} h_{\alpha \beta}^{\infty}$ one verifies the claim, and this proves the theorem.

\section{Proof of Theorems A ANd A ${ }^{\prime}$}

This section will be concerned with the proof of Theorems A and $A^{\prime}$. Note that by $\S 2.1$, we may treat the cases $\operatorname{dim} M=4$ and $\operatorname{dim} M>4$ on an equal footing, so we will assume $n=\operatorname{dim} N$ is arbitrary.

We will prove that conditions (i)-(vi) in Theorem $\mathrm{A}\left(\mathrm{A}^{\prime}\right)$ imply a bound on the sectional curvature $\left|K_{M}\right| \leq \Lambda^{2}$ of $M$. The result then follows from the 
Gromov compactness theorem. Suppose $\left|K_{M}\right|$ is not bounded on this set of Riemannian manifolds. Then there is a sequence of Riemannian $n$-manifolds $\left(M_{i}, g_{i}\right)$ such that (i)-(vi) hold uniformly for $\left(M_{i}, g_{i}\right)$ but

$$
\sup _{M_{i}}\left|K_{M_{i}}\right|=R_{i} \rightarrow \infty \quad \text { as } i \rightarrow \infty \text {. }
$$

It will be shown that this leads to a contradiction. Suppose the supremum above is achieved at $x_{i} \in M_{i}$ and consider the sequence of rescaled metrics $\left(M_{i}, h_{i}\right)$ with

$$
h_{i}=R_{i}^{1 / 2} \cdot g_{i}
$$

This sequence satisfies

$$
\begin{array}{ll}
\text { (1) } & \left|\operatorname{Ric}\left(h_{i}\right)\right| \leq c_{1} / R_{i} \rightarrow 0 \\
\text { (2) } & \sup _{r \leq \varepsilon R_{i}} r^{2(1-n / q)}\left[\int_{B(r)}\left|D^{2} \operatorname{Ric}\left(h_{i}\right)\right|\right]^{2 / q} \rightarrow 0 \\
\text { (3) } & \left|K\left(h_{i}\right)\right| \leq 1 \\
\text { (4) } & \left|K\left(h_{i}\right)\left(x_{i}\right)\right|=1 \\
\text { (5) } & \int_{M_{i}}\left|R\left(h_{i}\right)\right|^{n / 2} \leq c_{6} \\
\text { (6) } & l_{M_{i}}\left(h_{i}\right) \geq c_{3} R_{i} \rightarrow \infty \\
\text { (7) } & v(r) / r^{n}=\operatorname{vol}_{h_{i}} B_{x}(r) / r^{n} \geq c_{1} \\
\text { (8) } & \operatorname{inj}_{M_{i}}(x) \geq c_{0} .
\end{array}
$$

Here (1)-(6) follow from the bounds on $\left(M_{i}, g_{i}\right)$ and scaling properties, (7) follows from (2.3) and scale invariance (we assume $r \leq R_{i} / 2 \cdot \operatorname{vol}\left(M_{i}, g_{i}\right)$ ), and (8) follows from $\S 2.4$ via (3) and (7).

We wish to apply the Gromov compactness theorem (in local form) to the sequence $\left(M_{i}, g_{i}\right)$. However, a limit metric need only be $C^{1, \alpha}$ and we require some curvature properties, in particular, an analogue of (4), to apply to the limit. Thus, it is useful first to smooth the metrics and then to pass to a limit.

Let $h=h_{i}$ and consider the evolution equation of Hamilton [21]

$$
\partial h / \partial t=-2 \operatorname{Ric}(h)
$$

on $M=M_{i}$. It is shown in [3] that there is an $\varepsilon_{1}>0$, depending only on dimension (since $\left|K\left(h_{i}\right)\right| \leq 1$ ), such that solutions $h(t)$ to (3.4) exist for $0 \leq t \leq \varepsilon_{1}$, and

$$
\begin{aligned}
& \|h(t)-h\|_{C^{0}} \leq C(t), \quad \text { with } C(t) \rightarrow 0 \text { as } t \rightarrow 0, \\
& \left\|D^{k} R(t)\right\|_{C^{0}} \leq C\left(\frac{1}{t}, k\right), \quad k=1,2,3, \ldots, \\
& \|R(t)\|_{C^{0}} \leq 1 .
\end{aligned}
$$

One sees that the bounds $(3.3)(3),(7)$ and thus (8) remain valid for $h(t)$, for all $i, t$ with $t$ sufficiently small $t \leq \varepsilon_{1}$. We will show in a sequence of lemmas that the other bounds (except (2) and (6)) are also preserved, with (4) replaced 
by an analogue. In the following lemmas, we will suppress the dependence on $i$, but it is important to keep in mind that all estimates are independent of $i$. The "centerpoint" $x_{i} \in M_{i}$ will be denoted by $x_{0}$.

Lemma 3.1. For the metric $h=h(0)$, there are fixed positive constants $r_{0}, \delta_{0}$ such that

$$
\int_{B_{x_{0}}\left(r_{0}\right)}|R|^{2} \geq \delta_{0}
$$

Proof. Since $|R| \leq 1$, we may choose $r$ sufficiently small to apply Lemma 2.1 and obtain

$$
\sup _{B_{x_{0}}(r / 2)}|R|^{2} \leq C \cdot\left[\frac{1}{r^{2}} \int_{B_{x_{0}}(r)}|R|^{n / 2}+r^{2 \delta}\left(\int_{B_{x_{0}}(r)}\left|D^{2} \mathrm{Ric}\right|^{q / 2}\right)^{2 / q}\right],
$$

where $\delta=1-\frac{n}{q}$. By (3.3)(2), the second integral converges to zero as $i \rightarrow \infty$. On the other hand, by $(3.3)(4), \sup _{B_{x_{0}}(r / 2)}|R|^{2}=1$, so that

$$
\int_{B_{x_{0}}(r)}|R|^{n / 2} \geq c \cdot r^{2}
$$

Again, since $|R| \leq 1$, the result follows.

Lemma 3.2. For the metric $h=h(0)$, there is a constant $K$ such that

$$
\int_{B_{x}(1)}|\nabla R|^{2} \leq K
$$

for any $x \in M$.

Proof. From (2.5), it follows that one has the inequality

$$
\Delta|R|^{2} \geq-c_{1}\left|D^{2} \mathrm{Ric}\right| \cdot|R|-c_{2}|R|^{3}+|\nabla R|^{2} .
$$

Let $\zeta=\zeta(r)$ be a fixed cutoff function such that $\zeta(r) \equiv 1$ for $r \leq 1$ and $\zeta \equiv 0$ for $r \geq 2$. Multiply (3.8) by $\zeta$ and integrate by parts to obtain

$$
\int_{B_{x}(1)}|\nabla R|^{2} \leq \int_{M} \zeta \Delta|R|^{2}+c_{1} \int_{M} \zeta\left|D^{2} \mathrm{Ric}\right| \cdot|R|+c_{2} \int_{M} \zeta|R|^{3} .
$$

The latter two integrals on the right side are clearly bounded above. Noting that $\int_{M} \zeta \Delta|R|^{2}=\int_{M}|R|^{2} \Delta \zeta \leq \int_{M}|\Delta \zeta|$, this term may also be bounded from above by the local geometry of $M$.

Remark. Lemmas 3.1 and 3.2 are the only places where condition (ii) in Theorems $A$ and $A^{\prime}$ is required in the proof.

Lemma 3.3. There is a constant $L$ such that for $0 \leq t \leq \varepsilon_{1}$,

$$
\int_{B_{x}(1)}|\nabla R(t)|^{2} \leq L e^{L t}
$$


Proof. It follows from equation (13.3) of Hamilton [21] that one has the inequality

$$
\frac{d}{d t}|\nabla R|^{2} \leq \Delta|R|^{2}+c_{3}|\nabla R|^{2} \cdot|R| .
$$

Choose a locally finite cover of $M$ by geodesic balls $B_{p_{j}}\left(r_{1}\right)$ in the $h=h(0)$ metric, where $r_{1}<\frac{1}{2} \operatorname{inj}_{M}(h(0))$ and such that the balls $B_{p_{j}}\left(r_{1} / 2\right)$ also cover $M$. There is a $p_{1} \in\left\{p_{j}\right\}$ such that $\int_{B_{p_{1}}\left(r_{1}\right)}|\nabla R|^{2} \geq \int_{B_{p_{j}}\left(r_{1}\right)}|\nabla R|^{2}$, for all $j$. Further, since for any $D \subset M, \int_{D}|\nabla R(t)|^{2} d V_{t}$ is a continuous function in $t$, there is a $t_{1} \leq \varepsilon_{1}$ and $p_{1}$ such that, for all $j$,

$$
\int_{B_{p_{1}}\left(r_{1}\right)}|\nabla R(t)|^{2} d V_{t} \geq \int_{B_{p_{j}}\left(r_{1}\right)}|\nabla R(t)|^{2} d V_{t},
$$

for $t \leq t_{1}$. Let $\zeta=\zeta(r)$ be a fixed cutoff function with $\zeta(r) \equiv 1$ for $r \geq r_{1} / 2$ and $\zeta(r) \equiv 0$ for $r \geq 3 r_{1} / 4$. Multiply (3.10) by $\zeta$ and integrate by parts to obtain

$$
\begin{aligned}
\frac{d}{d t} \int_{B_{p_{1}}\left(r_{1}\right)} \zeta|\nabla R(t)|^{2} d V_{t} & =\int_{B_{p_{1}}\left(r_{1}\right)} \zeta \frac{d}{d t}|\nabla R(t)|^{2} d V_{t}+\int_{B_{p_{1}}\left(r_{1}\right)} \zeta|\nabla R(t)|^{2} \tau d V_{t} \\
& \leq \int_{B_{p_{1}}\left(r_{1}\right)}|\nabla R(t)|^{2}|\Delta \zeta| d V_{t}+c_{4} \int_{B_{p_{1}}\left(r_{1}\right)} \zeta|\nabla R(t)|^{2} d V_{t},
\end{aligned}
$$

where $\tau$ is the scalar curvature. One may bound $|\Delta \zeta| \leq c_{5}$ by a constant depending only on the local geometry, so that

$$
\begin{aligned}
\frac{d}{d t} \int_{B_{p_{1}}\left(r_{1}\right)} \zeta|\nabla R(t)|^{2} d V_{t} & \leq c_{6} \int_{B_{p_{1}}\left(r_{1}\right)}|\nabla R(t)|^{2} d V_{t} \\
& \leq c_{7} \int_{B_{p_{1}}\left(r_{1}\right)} \zeta|\nabla R(t)|^{2} d V_{t},
\end{aligned}
$$

where we have used (3.11) in the last inequality. Setting

$$
f(t)=\int_{B_{p_{1}}\left(r_{1}\right)} \zeta|\nabla R(t)|^{2} d V_{t},
$$

we obtain $f^{\prime} \leq c_{7} f$ for $t \leq t_{1}$. Thus, $f(t) \leq f(0) e^{c_{7} t} \leq K \cdot e^{c_{7} t}$ so that (3.9) holds for $t \leq t_{1}$. Repeating this argument with $t_{1}$ in place of $t_{0}=0$ and continuing by induction gives the result.

Lemma 3.4. For all $t \leq \varepsilon_{1}$ there is a constant $C$ such that

(i) $\quad \int_{B_{x_{0}}(1)}|R(t)|^{2} d V_{t} \geq C^{-1}$,

(ii) $\quad \int_{M}|R(t)|^{n / 2} d V_{t} \leq C$,

(iii) $\quad|\operatorname{Ric}(t)| \leq C \cdot|\operatorname{Ric}(h(0))|$. 
Proof. (i) Using equation (13.3) of Hamilton [21] again, one obtains the inequality

$$
\frac{d}{d t}|R|^{2} \geq \Delta|R|^{2}-2|\nabla R|^{2}-c_{3}|R|^{3}
$$

Choosing $\zeta$ as in Lemma 3.2 and integrating by parts gives

$$
\frac{d}{d t} \int_{B_{x_{0}}(1)} \zeta|R|^{2} d V \geq-\int_{B_{x_{0}}(1)}|\Delta \zeta|-2 \int_{B_{x_{0}}(1)}|\nabla R|^{2} \int_{B_{x_{0}}(1)}|R|^{3}
$$

The result then follows from Lemmas 3.1 and 3.3.

(ii) From equation (13.3) of [21] one obtains

$$
\frac{d}{d t}|R|^{2} \leq \Delta|R|^{2}+c_{3}|R|^{3}
$$

from which one deduces

$$
\frac{d}{d t}|R|^{n / 2} \leq \Delta|R|^{n / 2}+c_{3}|R|^{(n+2) / 2} .
$$

Integrating this inequality over $(M, h(t))$, and using $|R| \leq 1$ and (3.3)(5) gives the result.

(iii) From Corollary 7.3 of [21], the Ricci curvature satisfies

$$
\frac{d}{d t}|\operatorname{Ric}|^{2} \leq \Delta|\operatorname{Ric}|^{2}+c_{3}|R| \cdot|\operatorname{Ric}|^{3}
$$

Taking into account that $|R| \leq 1$, the result follows by applying the maximum principle on $M$, cf. $[3,21]$.

Returning now to the sequence of metrics $\left\{h_{i}\right\}$ on $M_{i}$, the above results show that there are metrics $h_{i}(t), t \leq \varepsilon_{1}$, on $M_{i}$, such that

$$
\begin{aligned}
\text { (i) } & \left\|h_{i}(t)-h_{i}\right\|_{C^{0}} \leq C(t), \\
\text { (ii) } & \left\|\nabla^{k} R_{i}(t)\right\|_{C^{0}} \leq C\left(\frac{1}{t}, k\right), \\
\text { (iii) } & \left\|R_{i}(t)\right\|_{C^{0}} \leq 1, \\
\text { (iv) } & \int_{M_{i}}\left|R_{i}(t)\right|^{n / 2} \leq C, \\
\text { (v) } & \int_{B_{x_{i}}(1)}\left|R_{i}(t)\right|^{2} \geq C^{-1}, \\
\text { (vi) } & \operatorname{vol}_{h_{i}(t)} B(r) \cdot r^{-n} \geq C^{-1}, \\
\text { (vii) } & \operatorname{inj}_{h_{i}(t)} M_{i} \geq C^{-1} .
\end{aligned}
$$

We now apply the Gromov compactness theorem to the pointed manifolds $\left(M_{i}, x_{i}, h_{i}(t)\right)$. By Theorem 2.2, the sequence $\left(B_{x_{i}}(R), h_{i}(t)\right)$ of Riemannian manifolds has a subsequence which converges, uniformly in the $C^{k, \alpha}$ topology for any $k$, to a $C^{\infty}$ Riemannian manifold $\left(B_{x_{\infty}}(R), h_{\infty}(t)\right)$, provided $t>$ 0 . By choosing a sequence $\left\{R_{j}\right\} \rightarrow \infty$ and taking a diagonal subsequence, it follows that a subsequence of $\left(M_{i}, x_{i}, h_{i}(t)\right)$ converges, smoothly on compact 
subsets, to a $C^{\infty}$ Riemannian manifold $N$, with smooth metric $h_{N}(t), 0<$ $t<\varepsilon_{1}$, satisfying

$$
\begin{aligned}
\text { (i) } & \operatorname{Ric}_{N} \equiv 0, \\
\text { (ii) } & \int_{N}|R|^{n / 2}<\infty, \\
\text { (iii) } & v(r) / r^{n} \geq C, \\
\text { (iv) } & \int_{B_{z}(1)}|R|^{2}>0
\end{aligned}
$$

for some point $z \in N$. We note that (3.14)(i) follows from (3.3)(1) and (3.12)(iii).

Let us indicate briefly how the result (3.14) leads to the required contradiction in the proof of Theorems $A$ and $A^{\prime}$. We will show below, and this is the crucial result, that a complete Riemannian manifold $V$ satisfying (3.14)(i)(iii) is asymptotic to a cone on a spherical space form $S^{n-1} / \Gamma$. Further, if $V$ is simply connected at infinity, then $V$ is flat. Below, we will use the bound (iii) in Theorems $\mathrm{A}$ and $\mathrm{A}^{\prime}$, or equivalently $(3.3)(6)$, to show that $N$, constructed above, is simply connected at infinity. Thus, $N$ must be flat, contradicting (3.14)(iv).

Theorem 3.5. Let $V$ be a complete, noncompact Riemannian manifold satisfying (3.14)(i)-(iii). Then given $z \in V$, there is an $R_{0}$ such that $V \backslash B_{z}\left(R_{0}\right)$ is diffeomorphic to $\left(R_{0}, \infty\right) \times S^{n-1}(1) / \Gamma$, where $S^{n-1}(1) / \Gamma$ is a spherical space form. Further, $V$ is asymptotically flat, i.e., the metric on $V$ approaches the flat metric on $\left(R_{0}, \infty\right) \times S^{n-1}(1) / \Gamma$ at a rate $O\left(1 / r^{2}\right)$.

If $V$ is simply connected at infinity, i.e., $\Gamma=\{e\}$, then $V$ is isometric to $\mathbf{R}^{n}$.

Proof. First note that from Bishop's inequality (§2.5) and (3.14)(iii), there is a constant $c_{0}$ such that $c_{0}^{-1} \leq v(r) / r^{n} \leq c_{0}$. It follows from an observation of Yau [40], based on the methods of Croke [10], that there is a global Sobolev constant $c_{s}$ for functions of compact support on $V$, i.e.,

$$
\|f\|_{2 n /(n-2)} \leq c_{s}\|d f\|_{2} \text {. }
$$

Consider now the annulus $A\left(\frac{1}{2} r, 2 r\right)=\left\{x \in V: \frac{1}{2} r \leq \operatorname{dist}(x, z) \leq 2 r\right\}$. Since $\int_{V}|R|^{n / 2}<\infty$, we have $\int_{A(r / 2,2 r)}|R|^{n / 2} \rightarrow 0$ as $r \rightarrow \infty$. We may apply Lemma 2.1 and find there is an $R_{0}>0$ such that, for all $r \geq R_{0}$,

$$
\sup _{S(r)}|R| \leq \frac{c}{r^{2}} \int_{A(r / 2,2 r)}|R|^{n / 2} \equiv \frac{\varepsilon(r)}{r^{2}}
$$

where $\varepsilon(r) \rightarrow 0$ as $r \rightarrow 0$. In other words, the curvature of $V$ decays faster than quadratically. It follows that the rescaled Riemannian manifolds $\left(A^{r}, g^{r}\right)=\left(A(r / 2,2 r), g / r^{2}\right)$ have sectional curvature satisfying

$$
\sup _{A^{r}}\left|R^{r}\right| \leq 2 \varepsilon(r) \rightarrow 0
$$


Clearly, by Bishop's inequality, vol $A^{r} \leq c_{6}$. If $B^{r}(s)$ is a geodesic ball of radius $s$ in $A^{r}$, then

$$
\begin{aligned}
\operatorname{vol} B^{r}(s) & =\frac{\operatorname{vol} B(r s)}{r^{n}}=s^{n} \cdot \frac{\operatorname{vol} B(r s)}{(r s)^{n}} \\
& \geq s^{n} \lim _{s \rightarrow \infty} \frac{\operatorname{vol} B(r s)}{(r s)^{n}}=c_{0} s^{n} .
\end{aligned}
$$

Note also that the diameter of $A^{r}$ is bounded above.

These results imply by $\S 2.4$ that there is a uniform lower bound on the injectivity radius $\operatorname{inj}^{r}(x)$ in the $g^{r}$ metric at each $x \in A^{r}$,

$$
\operatorname{inj}^{r}(x) \geq \delta_{0}>0 \text {. }
$$

We may now apply the Gromov compactness theorem of $\S 2.6$ to an arbitrary sequence of components of $\left(A^{r_{i}}, g^{r_{i}}\right)$. It follows that a subsequence converges, in the $C^{1, \alpha}$ topology, to a connected $C^{1, \alpha}$ Riemannian manifold $A^{\infty}=A^{\infty}\left(\frac{1}{2}, 2\right)$ of the same dimension. It is not difficult to verify that $A^{\infty}$ is flat. One way to do this is to use the smoothing procedure carried out above to obtain bounds on $\left|D^{k} R\right|$ and pass to a (different) limit $\left(A^{\infty}\right)^{\prime}$, which is then flat. However, more directly, as we will see in $\S 4$, since $V$ (and so $A^{r}$ ) is Einstein, there is a curvature bound of the form $\left|D^{k} R_{A^{r}}\right| \leq c\left(k,\left|R_{A^{r}}\right|, \operatorname{inj}_{A^{r}}\right)$, so that in fact $\left(A^{r_{i}}, g^{r_{i}}\right)$ converges smoothly to its limit $A^{\infty}$. By (3.15), it follows that $A^{\infty}$ is a smooth, flat Riemannian manifold.

Clearly, this process may be carried out with the manifolds $\left(A(r / n, n r), g / r^{2}\right)$ for any $n>1$ and by passing to subsequences, one obtains connected limit manifolds $A_{n}^{\infty}$ with $A_{m}^{\infty} \subset A_{n}^{\infty}$ if $m<n$. Taking a sequence $n_{i} \rightarrow \infty$, we obtain a limit flat connected, Riemannian manifold $B_{\infty}\left(=B_{\infty}(0, \infty)\right)$ such that $A_{n}^{\infty} \subset B_{\infty}$ for any $n$.

We claim that $B_{\infty}$ is the cone on a spherical space form $C\left(S^{n-1}(1) / \Gamma\right)-\{0\}$. First note that $B_{\infty}$ has a distinguished distance function $\rho$, namely the limit of the distance function $r(x)$ to $z$ on $A(r / n, n r)$. The function $\rho: B_{\infty} \rightarrow \mathbf{R}^{+}$ is a Lipschitz function, with Lipschitz constant 1 , with $|\nabla \rho|=1$ a.e. Let $C(s)=\left\{x \in B_{\infty}: \rho(x)=s\right\}$ so that $C(s)$ is the limit of a sequence of geodesic spheres $\left(S_{z}\left(r_{i} s\right), g^{r_{i}}\right)$ in $V$. Thus, $\{C(s)\}$ is a family of equidistants in $B_{\infty}$, i.e., $\operatorname{dist}_{B_{\infty}}(C(s), C(t))=|s-t|$, with $|\rho(x)-\rho(y)|=|s-t|$ for $x \in C(s)$, $y \in C(t)$. We claim that $\operatorname{diam}_{B_{\infty}} C(s) \rightarrow 0$ as $s \rightarrow 0$. To see this, let $D\left(\frac{s}{2}\right)=$ $\left\{x \in B_{\infty}: \operatorname{dist}(x, C(s)) \leq \frac{s}{2}\right\}$ and note that vol $D\left(\frac{s}{2}\right) \leq c_{1} \cdot s^{n}$. We may cover $D\left(\frac{s}{2}\right)$ by a finite number, say $Q$, of balls $B_{x_{i}}\left(\frac{s}{4}\right)$ in $B_{\infty}$ with $x_{i} \in D\left(\frac{s}{2}\right)$ such that the balls $B_{x_{i}}\left(\frac{s}{8}\right)$ are disjoint. Then $\operatorname{vol} B_{x_{i}}\left(\frac{s}{8}\right) \geq c_{2} \cdot s^{n}$, so that

$$
Q c_{2} s^{n} \leq \operatorname{vol} D(s) \leq c_{3} s^{n} \text {. }
$$

Thus, $Q$ is bounded from above by a constant independent of $s$. In particular, any two points in $C(s)$ may be joined by a broken geodesic in $D\left(\frac{s}{2}\right)$ of length $\leq c_{4} \cdot Q \cdot s$. 
It follows that if we attach a point 0 to $B_{\infty}$ via $\rho$, then $\bar{B}_{\infty}=B_{\infty} \cup\{0\}$ is a complete length space in the sense of Gromov [20, 1.7]. In particular, $\rho$ extends continuously over $\{0\}$ and represents distance to $\{0\}$ in $\bar{B}_{\infty}$. Any point $x \in C(1)$ may be joined to 0 by a minimizing geodesic $\gamma_{x}$ (not necessarily unique), such that $\rho\left(\gamma_{x}(t)\right)=t$.

Let $\widetilde{B}_{\infty}$ be the universal cover of $B_{\infty}$. Since $\widetilde{B}_{\infty}$ is flat and simply connected, the developing map $D: \widetilde{B}_{\infty} \rightarrow \mathbf{R}^{n}$ is an isometric immersion. In particular, $D$ maps geodesics in $\widetilde{B}_{\infty}$ to geodesics (straight line segments) in $\mathbf{R}^{n}$. Choose a geodesic $\gamma_{x}$ in $B_{\infty}$ as above and let $\tilde{\gamma}_{x}$ be a lift of $\gamma_{x}$ to $\widetilde{B}_{\infty}$. Then $D\left(\tilde{\gamma}_{x}\right)$ is a line segment in $\mathbf{R}^{n}$ which we may normalize to start at $0 \in \mathbf{R}^{n}$. Let $y \in C(1)$ and choose points $p_{i} \in C\left(s_{i}\right)$, with $s_{i} \rightarrow 0$, and curves $\sigma_{i}, \sigma$ joining $p_{i}$ to $\gamma_{x} \cap C\left(s_{i}\right)$ and $x$ to $y$, respectively. Let $\gamma_{y}(i)$ be minimizing geodesics joining $p_{i}$ to $y$ in the homotopy class of the curves $\sigma \circ \gamma_{x} \circ \sigma_{i}$. Then a subsequence of the curves $\gamma_{y}(i)$ converges to a minimizing geodesic $\gamma_{y}$ joining $0 \in B_{\infty}$ and $y \in C(1)$. Let $\tilde{\gamma}_{y}$ be the lift of $\gamma_{y}$ to $\widetilde{B}_{\infty}$ corresponding to $\tilde{\gamma}_{x}$. Since $\operatorname{dist}\left(\gamma_{x}(t), \gamma_{y}(t)\right) \rightarrow 0$, as $t \rightarrow 0$, $\operatorname{dist}\left(\tilde{\gamma}_{x}(t), \tilde{\gamma}_{y}(t)\right) \rightarrow 0$ also, so that $\operatorname{dist}_{\mathbf{R}^{n}}\left(D\left(\tilde{\gamma}_{x}(t)\right), D\left(\tilde{\gamma}_{y}(t)\right)\right) \rightarrow 0$.

If $\Sigma(s)$ is the inverse image of $C(s)$ under $\Pi: \widetilde{B}_{\infty} \rightarrow B_{\infty}$, then we see that the developing map $D$ maps $\Sigma(s)$ into $S(s)$, the sphere of radius $s$ about 0 in $\mathbf{R}^{n}$. It is clear that $D$ is an isometric immersion of $\Sigma(s)$ onto a domain in $S(s)$, and since $\Sigma(s)$ is complete, $D$ maps $\Sigma(s)$ onto $S(s)$. Thus, $D$ is a covering map and since $n>2$, it follows that $D$ is a diffeomorphism and thus an isometry.

It follows that $\widetilde{B}_{\infty}$ is isometric to $\mathbf{R}^{n}-\{0\}$ and $\Gamma=\pi_{1}\left(B_{\infty}\right)$ acts by isometries on $\mathbf{R}^{n}-\{0\}$ and thus on $\mathbf{R}^{n}$ fixing $\{0\}$. So $\Gamma$ acts freely and isometrically on $S^{n-1}(1)$ so that $B_{\infty}$ is isometric to $\mathbf{R}^{n}-\{0\} / \Gamma=C\left(S^{n-1} / \Gamma\right)-\{0\}$, as claimed. The manifolds $A_{n}^{\infty} \subset B_{\infty}$ are the domains $A_{n}^{\infty}=\left\{x \in B_{\infty}: \frac{1}{n} \leq\right.$ $\rho(x) \leq n\}$, so that $A_{n}^{\infty}$ is isometric to the truncated cone on $S^{n-1} / \Gamma$. In particular, by the Gromov compactness theorem, the original manifolds $\left(A^{r_{i}}, g\right)$ have subsequences which are diffeomorphic to $\left(\frac{1}{2}, 2\right) \times S^{n-1}(1) / \Gamma$, for some $\Gamma$.

We see there is an $R_{0}>0$ such that, for all $r \geq R_{0}, A(r / 2,2 r)$ is diffeomorphic to $\left(\frac{1}{2}, 2\right) \times S^{n-1} / \Gamma$, where $\Gamma$ may possibly depend on $r$. However, since the convergence $A^{r} \rightarrow A^{\infty}$ is smooth, we now see that $|\nabla \rho(x)|>0$, for $\rho(x) \geq R_{0}$, where $\rho$ is a smooth approximation of the distance function $r$. Since $\left(\frac{1}{2}, 2\right) \times S^{n-1} / \Gamma$ and $(2,4) \times S^{n-1} / \Gamma^{\prime}$ are diffeomorphic only if $\Gamma=\Gamma^{\prime}$, we have $A\left(R_{0}, \infty\right)$ is diffeomorphic to $\left(R_{0}, \infty\right) \times S^{n-1} / \Gamma$. The volume growth assumption (3.14)(iii), together with the Cheeger-Gromoll splitting theorem, implies that $A\left(R_{0}, \infty\right)$ is connected.

This completes the first part of Theorem 3.5. To prove the second part, if $V$ is simply connected at infinity, then $\Gamma=\{e\}$. However, the arguments above 
imply

$$
\begin{aligned}
\lim _{r \rightarrow \infty} \frac{\operatorname{vol} B_{z}(r)}{r^{n}} & \geq \lim _{r \rightarrow \infty} \operatorname{vol} A^{r}\left(\frac{1}{m} r, r\right) \\
& =\operatorname{vol} B^{n}(1)-\operatorname{vol} B^{n}\left(\frac{1}{m}\right),
\end{aligned}
$$

for any $m>1$, where $B^{n}(r)$ is the Euclidean ball of radius $r$. Thus,

$$
\lim _{r \rightarrow \infty} \frac{\operatorname{vol} B_{z}(r)}{r^{n}} \geq \omega_{n}
$$

so that the result follows from Bishop's inequality ( $\$ 2.5)$.

We may now complete the proof of Theorems $\mathrm{A}$ and $\mathrm{A}^{\prime}$. Recall that prior to Theorem 3.5, we constructed a complete, noncompact Riemannian manifold $N$ satisfying (3.14)(i)-(iv). By Theorem 3.5, it follows that $N$ is diffeomorphic to the interior of a compact manifold $N$ with boundary $\partial N=S^{n-1} / \Gamma$.

So far, we have not used condition (iii) in Theorems $A$ and $A^{\prime}$, and we now use it to prove that $\Gamma=\{e\}$. First, from (3.14)(iii) and Proposition 1.2 of [1], it follows that $\pi_{1}(N)$ is a finite group. If we prove $\Gamma=\{e\}$ for $\tilde{N}$, so that $\tilde{N}=\mathbf{R}^{n}$, then clearly $N=\mathbf{R}^{n}$ also, since a finite group cannot act freely on $\mathbf{R}^{n}$. Thus, we may assume $\pi_{1}(N)=\{e\}$.

Recall that the manifold $N$ is constructed as a limit $\left(M_{i}, h_{i}(t)\right)$ with $0<t \leq$ $\varepsilon_{1}$. The manifolds $\left(M_{i}, h_{i}(t)\right)$ are close, in the Lipschitz topology, to the (unsmoothed) manifolds $\left(M_{i}, h_{i}\right), h_{i}=h_{i}(0)$. Note that, by Gromov's compactness theorem, a subsequence of $\left\{\left(M_{i}, h_{i}\right)\right\}$ converges, uniformly in the $C^{1, \alpha}$ topology on compact sets, to a complete Riemannian manifold $\left(M_{\infty}, h_{\infty}\right)$, with $C^{1, \alpha}$ metric $h_{\infty}$. In particular, geodesics in $\left(M_{i}, h_{i}\right)$ converge, uniformly on compact sets, to geodesics in $\left(M_{\infty}, h_{\infty}\right)$. Clearly, $\left(M_{\infty}, h_{\infty}\right)$ and $\left(N, h_{\infty}(t)\right)$ are close in the Lipschitz topology, so in particular they are diffeomorphic.

It follows that there is a sequence of domains $B_{i}=B_{x_{i}}(R) \subset\left(M_{i}, h_{i}\right)$, for an arbitrary but fixed $R$, such that $B_{i}$ is diffeomorphic, and metrically close, to $B_{z}(R) \subset\left(N, h_{N}(t)\right)$, for $i$ sufficiently large. In particular, $\partial B_{i} \approx S^{n-1} / \Gamma$ and since $B_{z}(R) \subset\left(N, h_{N}(t)\right)$ is a strictly convex domain, for $R$ large, $B_{i} \subset$ $\left(M_{i}, h_{i}\right)$ is also (for $i$ sufficiently large). Now there is an $R_{0}>0$ such that if $B_{0}=B_{x_{i}}\left(R_{0}\right) \subset B_{i}$, then $\pi_{1}\left(B_{i}-B_{0}\right)=\pi_{1}\left(\partial B_{i}\right)=\Gamma$. Suppose $\Gamma \neq\{e\}$. Fix a point $p=p_{i} \in \partial B_{i}$ and let $\gamma=\gamma_{i}$ be a curve of shortest length in $B_{i}-B_{0}$ representing a nonzero class in $\pi_{1}\left(B_{i}-B_{0}\right)$. We claim that if $|\Gamma|>3$, then $\gamma$ does not intersect $B_{0}$, and thus $\gamma$ is a geodesic loop in $B_{i}-B_{0}$ (since $\partial B_{i}$ is convex). To see this, if $\gamma \cap B_{0} \neq \varnothing$, then for $R_{0}$ sufficiently large, and $R \gg R_{0}, l(\gamma) \approx 2\left(R-R_{0}\right)$, since the metric on $B_{i}-B_{0}$ is close to the flat metric on $A\left(R_{0}, R\right) \subset C\left(S^{n-1} / \Gamma\right)$. However, since the metric on $\partial B_{i}$ is close to the canonical metric on $S^{n-1}(R) / \Gamma$, there is a curve $\sigma$ in $\partial B_{i}$ of length $l(\sigma) \approx 2 \pi R /|\Gamma|$. Since $2 \pi /|\Gamma|<2$ if $|\Gamma|>3$, this shows $\gamma \cap B_{0}=\varnothing$, for $R_{0}$, $R$, and $i$ sufficiently large. 
Thus, in this case, $B_{i}$ contains geodesic loops $\gamma=\gamma_{i}$ of a bounded length $l\left(\gamma_{i}\right) \approx 2 \pi /|\Gamma| \cdot R$. Since $B_{i}$ is simply connected, these geodesic loops are inessential. However, by (3.3)(6), the length of an inessential geodesic loop in $\left(M_{i}, h_{i}\right)$ (and thus in a finite cover of $\left.M_{i}\right)$, satisfies

$$
l_{M_{i}}\left(h_{i}\right) \geq c_{3} \cdot R_{i} \rightarrow \infty, \quad \text { as } i \rightarrow \infty .
$$

This contradiction shows that we must have $|\Gamma| \leq 3$. In this case, we may argue as follows. By (3.17), and Morse theory for the length functional of the $h_{i}$ metric, it follows that $\pi_{k}\left(\Omega^{t_{i}}\right)=0$ for all $k$, where $t_{i}=c_{3} \cdot R_{i}$ and $\Omega^{t_{i}}$ is the space of loops of length $<t_{i}$ in the $h_{i}$ metric. Since the metrics $h_{i}$ converge smoothly to the metric $h_{\infty}$, the length functional converges also and it follows, for instance from the minimax characterization, that $\pi_{k}(\Omega)=0$ for all $k$. (There are no critical points of the length functional of $h_{\infty}$ associated to any homotopy class $S^{k} \rightarrow \Omega$.) Thus, $\Omega$ and so $B_{i} \approx B$ is contractible. An elementary exact homology sequence argument for the pair $(B, \partial B)$, cf. also Lemma 6.3 , shows that $\partial B \approx S^{n-1} / \Gamma$ is a homology sphere. Since $|\Gamma| \leq 3$, this is only possible if $\Gamma=\{e\}$, as required.

Theorem 3.5 now implies that $N$ is flat, which contradicts property (3.14)(iv) of $N$. This completes the proof of Theorems $\mathrm{A}$ and $\mathrm{A}^{\prime}(\mathrm{I})$. To prove $\mathrm{A}^{\prime}$ (II), note that we may take the oriented double cover of the manifold $N$ satisfying (3.14)(i)-(iv) and obtain an oriented manifold, call it $N$ again, satisfying (3.14)(i)-(iv). Now by Theorem 3.5, $N$ has a well-defined boundary $S^{n-1} / \Gamma$ at infinity. If $n$ is odd, $S^{n-1}$ is an even-dimensional sphere, so the only space forms are $\Gamma=\{e\}$ or $\Gamma=\mathbf{Z}_{2}$. Since $\partial N$ carries a canonical orientation, and $\mathbf{R P}^{n-1}$ is nonorientable if $n$ is odd, this case is ruled out. Thus, $N$ is simply connected at infinity and the arguments above complete the proof.

Remarks. (1) The condition $l_{M} \geq c$ in Theorems A and $\mathrm{A}^{\prime}$ could be dropped if the hypotheses of Theorem 3.5 implied that $V$ is simply connected at infinity, and thus flat. However, this is false in even dimensions. In fact, a number of authors, $[7,13,22]$ among others, have produced examples of Ricci-flat metrics on simply connected 4-manifolds, which are asymptotic to lens spaces at infinity, and so in particular are not flat. These are examples of gravitational instantons, or gravitational analogues of the self-dual Yang-Mills fields on $\mathbf{R}^{4}$. In higher dimensions, Calabi [7] has produced examples of Ricci-flat Kahler metrics on the canonical line bundle over $\mathbf{C P}^{n-1}$. At infinity, these manifolds have the topology of $S^{2 n-1} / \mathbf{Z}_{n}$, and one may verify that their curvature tensor is in fact in $L^{2}$ for all $n$.

(2) For the proof of Theorem A under the assumption $\mathscr{A}_{M} \geq a>0$, we refer to the remark following the proof of Lemma 6.3.

(3) It would be interesting to know if the bound on $\int_{M}|R|^{n / 2}$ in Theorem $\mathrm{A}^{\prime}$ can be replaced by a bound on a weaker curvature integral. For instance, is it 
sufficient to bound $\sup _{r>0} r^{4-n} \int_{B(r)}|R|^{2}$ ? If $M$ is a Kahler-Einstein manifold, then this integral is related to topological invariants of $M$.

\section{Proof of Corollary B}

In this section, we will prove the parts of Corollary B. For the most part, the results follow in a straightforward way from Theorems $\mathrm{A}$ and $\mathrm{A}^{\prime}$.

If $(M, g)$ is an Einstein manifold, then $\mathrm{Ric}_{M}=\lambda g$ and we will assume in this section that the metric on $M$ is normalized so that $\lambda=-1,0$, or +1 . Since $D g=0$, we have $D$ Ric $=0$ so that condition (ii) in Theorems A and $\mathrm{A}^{\prime}$ is trivially satisfied.

(1)(a) If $\operatorname{Ric}_{M}=+1 g$, then $\operatorname{diam}_{M} \leq \pi$ by Myer's theorem. Thus, by Theorem A, the space $\mathscr{E}^{+1}\left(l_{M}, v_{M}, b_{2}(M)\right)$ of +1 Einstein metrics on compact 4-manifolds such that $l_{M} \geq c_{1}, \operatorname{vol}_{M} \geq c_{2}, b_{2}(M) \leq c_{3}$ is $C^{1,1}$ compact. Further, by the proof of Theorem $\mathrm{A}$, the sectional curvature $\left|K_{M}\right|$ is uniformly bounded on $\mathscr{E}^{+1}\left(l_{M}, v_{M}, b_{2}(M)\right)$. By Cheeger's theorem $(\S 2.4)$, there is a uniform lower bound on the injectivity radius.

Thus, we have uniform lower bounds for the size of harmonic balls for metrics $(M, g)$ in $\mathscr{E}^{+1}\left(l_{M}, v_{M}, b_{2}(M)\right)$, and also uniform bounds $\|g\|_{1, \alpha}$ for the metric tensor in these coordinates (cf. Fact of §2.6). Now in harmonic coordinates, the Einstein equation is an elliptic system of partial differential equations

$$
-g^{r s} \frac{\partial g_{i j}}{\partial x^{r} \partial x^{s}}+g^{r s}\left[\frac{\partial^{2} g_{r i}}{\partial x^{s} \partial x^{j}}+\frac{\partial^{2} g_{r i}}{\partial x^{s} \partial x^{i}}-\frac{\partial^{2} g_{r s}}{\partial x^{i} \partial x^{j}}\right]+\cdots=2 \lambda g_{i j},
$$

where the dots indicate lower order terms involving only one derivative of the metric, cf. [11]. It follows from standard elliptic theory [28] that one has bounds

$$
\|g\|_{k, \alpha} \leq C\left(k,\|g\|_{1, \alpha}\right)
$$

so that all covariant derivatives of the curvature tensor $D^{k} R$ have uniform bounds on $\mathscr{E}^{+1}\left(l_{M}, v_{M}, b_{2}(M)\right)$. By the Gromov compactness theorem (Theorem 2.2), we see that $\mathscr{E}^{+1}\left(l_{M}, v_{M}, b_{2}(M)\right)$ is thus compact in the $C^{k, \alpha}$ topology for any $k$.

(1)(b) Let $D$ be a connected component of the moduli space of Einstein metrics on a compact manifold $M$. It is well known that, first, tr Ric is constant on $D$, and, secondly, that the volume function

$$
\operatorname{vol}\left(g_{0}\right)=\operatorname{vol}: D \rightarrow \mathbf{R}
$$

is a constant function on $D$ [5]. In particular, the volume is bounded below on $D$ so the result follows from (1)(a).

(2), (3) These results follow in exactly the same way as in (1) above. 
(4) We argue by contradiction. If the statement were false, there would exist a sequence $\left(M_{i}, g_{i}\right)$ satisfying the required bounds such that

$$
\int_{M_{i}}\left|\operatorname{Ric}\left(g_{i}\right)-\lambda g_{i}\right| \rightarrow 0,
$$

but the $M_{i}$ have no Einstein metric Ric $=\lambda g$ for all $i$. However, the bounds on $\left(M_{i}, g_{i}\right)$ imply by Theorems $\mathrm{A}$ and $\mathrm{A}^{\prime}$ that the sectional curvature on $\left(M_{i}, g_{i}\right),\left|K\left(g_{i}\right)\right|$ is uniformly bounded. Further, the metrics $g_{i}$ have a $C^{1, \alpha}$ convergent subsequence to a $C^{1, \alpha}$ metric $g$ on a smooth manifold $M$. Clearly, $M$ is diffeomorphic to $M_{i^{\prime}}$ for a subsequence $i^{\prime} \rightarrow \infty$. Note, however, that $g$ is a weak $\left(C^{1, \alpha}\right)$ solution of the Einstein equation (4.1). The regularity theory for elliptic systems [28] then implies that $g$ is smooth and satisfies the Einstein equation $\operatorname{Ric}(g)=\lambda g$. This contradicts the assumption, which proves the first statement. The other statements are proved in the same way.

\section{CONVERGENCE OF EINSTEIN METRICS}

This section will be devoted to the proof of Theorem C. The proof uses a number of methods and results from the previous sections. Some of these techniques are also used in [14, 29]; cf. also [33] for a very readable exposition. Let $\left(M_{i}, g_{i}\right)$ be a sequence of Einstein manifolds satisfying the conditions of Theorem C. It follows from $\S 2.2$ that one has uniform lower bounds for the Sobolev constants $c_{s}$ of $\left(M_{i}, g_{i}\right)$ and also for the growth of small geodesic balls $B_{x}(r) \subset M_{i}$, namely $v(r) / r^{n} \geq c_{0}$, by (2.3).

By a theorem of Gromov $[20,5.3],\left(M_{i}, g_{i}\right)$ has a subsequence which converges, in the Hausdorff topology, to a compact length space $\left(M_{\infty}, g_{\infty}\right)$. In particular, $\left(M_{\infty}, g_{\infty}\right)$ is a connected metric space, and there is a well-defined notion of geodesic balls in $M_{\infty}$ with the geodesic balls in $M_{i}$ (sub)converging to geodesic balls in $M_{\infty}$ in the Hausdorff topology. Further, the notion of length of a curve is well defined and between any two points in $M_{\infty}$, there is a minimizing geodesic.

Let $\varepsilon_{0}$ be the constant of Lemma 2.1, determined by the bounds Ric $_{M_{i}}=$ $\lambda_{i} g_{i}, \operatorname{vol}_{M_{i}} \geq c_{1}, \operatorname{diam}_{M_{i}} \leq c_{2}$ on $\left(M_{i}, g_{i}\right)$. We fix an $r>0$ but small and let $\left\{x_{k}\right\}=\left\{x_{k}^{i}\right\}$ be a maximal $\frac{r}{2}$ separated set in $M$. Thus, the geodesic balls $B_{x_{k}}\left(\frac{r}{4}\right)$ are disjoint, while the balls $B_{x_{k}}(r)$ form a cover of $M_{i}$. We let

$$
G^{r}=G_{i}^{r}=\bigcup\left\{B_{x_{k}}(r): \int_{B_{x_{k}}(2 r)}|R|^{n / 2}<\varepsilon_{0}\right\},
$$

where $R=R_{i}$ is the curvature tensor of $\left(M_{i}, g_{i}\right)$. Similarly, set

$$
B^{r}=B_{i}^{r}=\bigcup\left\{B_{x_{k}}(r): \int_{B_{x_{k}}(2 r)}|R|^{n / 2} \geq \varepsilon_{0}\right\} .
$$


Then $M_{i}=G_{i}^{r} \cup B_{i}^{r}$. Note that there is a bound on the number of balls $Q_{i}^{r}$ in $B_{i}^{r}$, independent of $i$ and $r$, namely,

$$
Q_{i}^{r} \leq \frac{c_{3}}{\varepsilon_{0}} \cdot m,
$$

where $c_{3}$ is the bound (iii) in Theorem $\mathrm{C}$ and $m$ is the maximal number of disjoint balls of radius $\frac{r}{2}$ in $M_{i}$ contained in a ball of radius $2 r$. By the (relative) Bishop comparison theorem, there is a uniform bound on $m$, independent of $i, r$.

Now, by Lemma 2.1, on each $G_{i}^{r}$ there is a uniform curvature bound $\left|R_{i}(x)\right|$ $\leq C\left(\operatorname{dist}\left(x, B_{i}^{r}\right)\right) \leq C\left(r^{-1}\right)$. Clearly, vol $G_{i}^{r}$ is uniformly bounded above, since this holds for $\left(M_{i}, g_{i}\right)$. From the bound above, on the volume growth of small geodesic balls, and from $\S 2.4$, it follows that the injectivity radius of $M_{i}$ at each $x \in G_{i}^{r}$ has a uniform lower bound, depending only on $r$ and the bounds on $\left(M_{i}, g_{i}\right)$. Thus, the regularity result (4.2) implies a uniform bound on the covariant derivatives $\left|D^{k} R\right|(x)$ for $x \in G_{i}^{r}$.

By Theorem 2.2, for $r>0$ fixed, a subsequence of $\left\{G_{i}^{r}\right\}$ converges, in the $C^{k, \alpha}$ topology on compact sets, to a smooth Riemannian manifold $G^{r}$ with Einstein metric $g_{\infty}^{r}$. In particular, $G^{r}$ and $G_{i}^{r}$ are diffeomorphic for $i$ sufficiently large (in the subsequence) so that there are smooth embeddings $F_{i}^{r}: G^{r} \rightarrow M_{i}$ such that $\left(F_{i}^{r}\right)^{*} g_{i}$ converges uniformly in the $C^{k, \alpha}$ topology to $g_{\infty}^{r}$ on $G^{r}$.

We now choose a sequence $\left\{r_{j}\right\} \rightarrow 0$ with $r_{j+1}<\frac{1}{2} r_{j}$ and perform the above construction for each $j$. Let $G_{i}\left(r_{l}\right)=\left\{x \in M_{i}: x \in G_{i}^{j}\right.$, for some $\left.j \leq l\right\}$ so that one has inclusions

$$
G_{i}\left(r_{1}\right) \subset G_{i}\left(r_{2}\right) \subset \cdots \subset M_{i} .
$$

By the argument above, each $\left\{G_{i}\left(r_{l}\right)\right\}$, for $l$ fixed, has a smoothly convergent subsequence to a limit $G\left(r_{l}\right)$. Clearly, $G\left(r_{l}\right) \subset G\left(r_{l+1}\right)$ and we set

$$
G=\bigcup_{1}^{\infty} G\left(r_{l}\right),
$$

with the induced Riemannian metric $g_{\infty}$. In particular, $\left(G, g_{\infty}\right)$ is a smooth Einstein manifold. It follows that there are smooth embeddings $F_{i}^{l}: G\left(r_{l}\right) \rightarrow$ $M_{i}$, for $i$ sufficiently large, such that $\left(F_{i}^{l}\right)^{*}\left(g_{i}\right)$ converges, smoothly in the $C^{k, \alpha}$ topology on $G\left(r_{l}\right)$, to the metric $g_{\infty}$.

Let $\bar{G}$ be the metric completion of $G$. We claim there is a finite set of points $\left\{p_{i}\right\}, i=1, \ldots, Q^{\prime}$, such that

$$
\bar{G}=G \cup\left\{p_{i}\right\} .
$$

To see this, we return to the sets $B_{i}^{r} \subset M_{i}$. Let $\left\{r_{j}\right\}$ be as above and recall from (5.1) that the cardinality of the number of balls in $B_{i}^{j} \subset M_{i}$ is uniformly 
bounded in $i, j$; by passing to subsequences, we may assume it is constant, say $Q$. Thus, there is a finite set of disjoint geodesic balls $\left\{B_{x_{k}}\left(Q \cdot r_{j}\right)\right\}_{i=1}^{Q^{\prime}}$, with $Q^{\prime} \leq Q$, which, together with $G_{i}\left(r_{j}\right)$, form a cover of $M_{i}$. In particular, every point of $M_{i}-G_{i}\left(r_{j}\right)$ is contained in a ball of diameter $\leq 2 \cdot Q r_{j}$. Using the embeddings $F_{i}^{l}: G\left(r_{l}\right) \rightarrow M_{i}$, we see that for any fixed $j$, and $i$ sufficiently large, arbitrarily large compact subsets of $G-G\left(r_{j}\right)$ are almost isometrically embedded into $Q^{\prime}$ disjoint balls of radius $\leq c \cdot r_{j}$. Taking the limit as $i \rightarrow \infty$, it follows that the Hausdorff distance between $\left(G-G\left(r_{j}\right), g_{\infty}\right)$ and the set of $Q^{\prime}$ distinct points $\left\{p_{i}\right\}$ is $\leq c \cdot r_{j}$. Letting $j \rightarrow \infty$, it follows that the Hausdorff distance between $\partial G=\bar{G}-G$ and $\left\{p_{i}\right\}$ is 0 , and thus these spaces are isometric. This gives (5.4).

It is now easy to see that a subsequence of $\left\{M_{i}\right\}$ converges to $\bar{G}$ in the Hausdorff topology. In fact, if $N=\left\{x_{k}\right\}$ is any $\varepsilon$-net in $\bar{G}$, with $x_{k} \notin\left\{p_{i}\right\}$, then the embeddings $F_{i}: G \rightarrow M_{i}$ give an $\varepsilon^{\prime}$-net $N_{i}$ in $M_{i}$ converging to $N$ in the Lipschitz topology (cf. $[20,3.5]$ ). Thus, $\bar{G}=M_{\infty}$, so that $\bar{G}$ is a complete length space, with length function $g_{\infty}$, which restricts to a smooth Einstein metric on $G$.

Definition. The points $\left\{p_{i}\right\}_{1}^{Q^{\prime}}$ are called the curvature singularities of $M_{\infty}$.

We now examine the structure, topological and metric, of the smooth manifold $N_{j}=B_{p_{j}}(r)-\left\{p_{j}\right\} \subset M_{\infty}$ for $r$ small, by essentially studying the tangent cones of $M_{\infty}$ at $p_{j}$. We will drop the subscript $j$ for convenience. First, note that there is a uniform lower bound for the Sobolev constant $c_{s}$ (2.2) for functions of compact support in $N$, since on any compact subset the convergence of $M_{i}$ is smooth and there is a uniform Sobolev constant for $\left\{M_{i}\right\}$. In particular, it follows that $v(s) / s^{n}=\operatorname{vol} B_{x}(s) / s^{n} \geq c_{0}$, for any geodesic ball $B_{x}(s) \subset N$.

Now $N$ is an Einstein manifold with $\int_{N}|R|^{n / 2}<\infty$. Let $r(x)$ be the distance function to $p$ in $M_{\infty}$ and let $N(s, t)=\{t \in N, s \leq r(x) \leq t\}$. It follows that

$$
\int_{N(s / 2,2 s)}|R|^{n / 2} \rightarrow 0 \text { as } s \rightarrow 0
$$

so that there is an $R_{0}$ such that for $s \geq R_{0}, \int_{N(s / 2,2 s)}|R|^{n / 2} \leq \varepsilon_{0}$, where $\varepsilon_{0}$ is the constant from Lemma 2.1. Thus, by Lemma 2.1, there is a function $\mu(s)$, with $\mu(s) \rightarrow 0$ as $s \rightarrow 0$ such that

$$
\sup _{S(s)}|R| \leq \mu(s) / s^{2}
$$

where $S(s)=\{x \in N: r(x)=s\}$.

Now we proceed exactly as in the proof of Theorems A and $\mathrm{A}^{\prime}$, but blowing the metric up instead of down. Namely, given any sequence $s_{i} \rightarrow 0$, the Riemannian manifolds $A\left(s_{i} / 2,2 s_{i}\right)=\left(N\left(s_{i} / 2,2 s_{i}\right), g_{\infty} / s_{i}^{2}\right)$ have sectional curvatures converging to zero by (5.5). Further, the volumes of geodesic balls in 
$A\left(s_{i} / 2,2 s_{i}\right)$ have upper and lower bounds $c_{0} \leq v(r) / r^{n} \leq c_{0}^{-1}$ and thus by $\S 2.4$, the injectivity radius of points $x \in A\left(s_{i} / 2,2 s_{i}\right)$ have a uniform lower bound. Let us estimate the number of components of $A\left(s_{i} / 2,2 s_{i}\right)$. If $\delta$ is a lower bound for the injectivity radius in $A\left(s_{i} / 2,2 s_{i}\right)$, consider a maximal $\frac{\delta}{2}$ separated set $\left\{x_{j}\right\}$ in $A\left(s_{i} / 2,2 s_{i}\right)$. Then the balls $B_{x_{j}}\left(\frac{\delta}{2}\right)$ are disjoint and the balls $B_{x_{j}}(\delta)$ cover $A\left(s_{i} / 2,2 s_{i}\right)$. From the above volume bounds, it follows that the cardinality of $\left\{x_{j}\right\}$ is uniformly bounded, independent of $i$. Clearly, points which lie in distinct components of $A\left(s_{i} / 2,2 s_{i}\right)$ are (more than) $\delta$ separated so that one obtains a uniform upper bound on the number of components of $A\left(s_{i} / 2,2 s_{i}\right)$. As in the proof of Corollary B(1)(a), there are uniform bounds on the covariant derivatives $\left|D^{k} R\right|$ of the curvature of the metric $g_{\infty} / s_{i}^{2}$ on $A\left(s_{i} / 2,2 s_{i}\right)$. It follows from Theorem 2.2 that a subsequence converges smoothly to a flat Riemannian manifold $A_{\infty}\left(\frac{1}{2}, 2\right)$ with a finite number of components.

This process may be carried out for $\left(N\left(s_{i} / n, n s_{i}\right), g_{\infty} / s_{i}^{2}\right)$, for any given $n$, and gives rise, by passing to a diagonal subsequence, to a flat Riemannian manifold $B_{\infty}$ with $A_{\infty}\left(\frac{1}{n}, n\right) \subset B_{\infty}$ for all $n$. The proof of Theorems A and $A^{\prime}$ shows that each component of $B_{\infty}$ is a cone on a spherical space form $S^{n-1}(1) / \Gamma$ and as before, it follows that each component of $N$ is diffeomorphic to $(0, r) \times S^{n-1} / \Gamma$.

We now show that $N=B_{p}(r)-p$ is connected for all $r$ small. In fact, we will show that $\partial B_{p}(r)=S_{p}(r)$ is connected. First, it is clear that $B_{p}(r)$ is connected. Since $M_{i} \rightarrow M_{\infty}$ in the Hausdorff topology, there are smooth connected domains $D_{i} \subset M_{i}$ with $D_{i} \rightarrow B_{p}(r)$ in the Hausdorff topology. Further, since the convergence $M_{i} \rightarrow M_{\infty}$ is smooth away from $p, \partial D_{i}=S_{i}$ converges to $S_{p}(r)$ in the Lipschitz topology. By the results above, $S_{p}(r)$ is convex, so that each component of $S_{i}$ is convex, i.e., has positive definite second fundamental form II w.r.t. the inward normal; in fact, II is approximately $\frac{1}{r} \mathrm{I}$ for $i$ large. We will suppose $S_{p}(r)$, and thus $\partial D_{i}$, is disconnected and we obtain a contradiction.

Let $\gamma$ be a geodesic in $D=D_{i}$ realizing the minimum distance between two distinct boundary components $C_{1}, C_{2}$ of $\partial D=\partial D_{i}$. A standard form of the second variational formula [6] then gives

$$
\mathrm{II}_{2}\left(E_{2}, E_{2}\right)+\mathrm{II}_{1}\left(E_{1}, E_{1}\right)=\int_{\gamma}\left|\nabla_{T} E\right|^{2}-K(E, T),
$$

where $\mathrm{II}_{i}$ is the second fundamental form of $C_{i}$ (w.r.t. the inward normal), $E$ is the Jacobi field along $\gamma$ determined by the boundary conditions $E(0)=E_{1}$, $E(l(\gamma))=E_{2}$, and $T$ is the unit tangent vector to $\gamma$. By the basic inequality for Jacobi fields (since there are no focal points of the normal exponential map of $\partial D_{i}$ along $\gamma$ ),

$$
\int_{\gamma}\left|\nabla_{T} E\right|^{2}-K(E, T) \leq \int_{\gamma}\left|\nabla_{T} X\right|^{2}-K(X, T)
$$


for any vector field $X$ along $\gamma$ with the same boundary conditions. Choose $X$ to be a parallel vector field along $\gamma$ with initial condition $E=E(0)$ a unit vector, and then sum (5.6) and (5.7) over an orthonormal basis of initial vectors. We then find

$$
H_{2}+H_{1} \leq-l(\gamma) \cdot \operatorname{Ric}_{M_{i}}
$$

where $H_{i}$ is the mean curvature of $C_{i}$ at the endpoints of $\gamma$. By the facts above, $H_{i} \approx(n-1) / r$, so that

$$
\frac{2(n-1)}{r} \lesssim-l(\gamma) \cdot \operatorname{Ric}_{M_{i}}
$$

for $i$ large. If $\mathrm{Ric}_{M_{i}}=0$ or +1 , this is clearly impossible. If $\mathrm{Ric}_{M_{i}}=-1$, this is also impossible for $r$ sufficiently small. It follows that $B_{p}(r)-p \subset M_{\infty}$ is connected and thus $B_{p}(r) \simeq B^{n}(1) / \Gamma$, the quotient of a ball in $\mathbf{R}^{n}$ by a finite group of orthogonal isometries.

Remark. Of course, it is possible that $\Gamma=\{e\}$, so that there may be curvature singularities on $M_{\infty}$, as defined in (5.4), which are regular or smooth points of $M_{\infty}$.

The arguments above prove that $M_{\infty}$ has the structure of an orbifold with a finite number of curvature singularity points, each having a punctured neighborhood which is diffeomorphic to a punctured cone on a spherical space form. We now examine the metric behavior of these singularities $(N, p) \subset M_{\infty}$. It follows from the work above that the manifolds $N\left((s / 2,2 s), g_{\infty} / r^{2}\right)$ converge, in the $C^{k}$ topology, to the flat metric $g_{0}$ on $S^{n-1} / \Gamma \times\left(\frac{1}{2}, 2\right)$, as $s \rightarrow 0$. In particular, there are $C^{k}$ diffeomorphisms $\phi_{s}: S^{n-1} / \Gamma \times\left(\frac{1}{2}, 2\right) \rightarrow N(s / 2,2 s)$ such that

$$
\left\|\phi_{s}^{*}\left(g_{\infty} / r^{2}\right)-g_{0}\right\|_{C^{k}}=\varepsilon(s),
$$

where $\varepsilon(s) \rightarrow 0$ as $s \rightarrow 0$. Further, the distance functions $r=\operatorname{dist}_{N}(x, p)$ and $r_{0}=\operatorname{dist}_{S^{n-1} / \Gamma \times(0, t)}(x, 0)$ converge, in the sense that $\left(\phi_{s}^{*}(r) / r_{0}\right)(x) \rightarrow 1$, as $s \rightarrow 0$. Thus,

$$
\phi_{s}^{*} g_{\infty}=\left(r \circ \phi_{s}\right)^{2} \cdot g_{0}+\left(r \circ \phi_{s}\right)^{2} \mu(s)=\frac{\left(r \circ \phi_{s}\right)^{2}}{r_{0}^{2}} r_{0}^{2} \cdot g_{0}+\left(r \circ \phi_{s}\right)^{2} \mu(s) \text {. }
$$

This says that if we view $\phi_{s}^{*} g_{\infty}$ as a metric on $S^{n-1} / \Gamma \times(s / 2,2 s)$, then

$$
\phi_{s}^{*} g_{\infty}=\frac{\left(r \circ \phi_{s}\right)^{2}}{r_{0}^{2}} g_{0}+\left(r \circ \phi_{s}\right)^{2} \mu(s),
$$

where $\mu(s) \rightarrow 0$, as $s \rightarrow 0$. Now the diffeomorphisms $\phi_{s}$ are not unique. However, we may view $g_{s}=g_{\infty \mid N(s / 2,2 s)}$ as a smooth curve in the space of metrics on a fixed manifold, say $N\left(s_{0} / 2,2 s_{0}\right)$. It is then clear that the diffeomorphisms $\phi_{s}$ may be chosen to depend smoothly on $s$, satisfying (5.8). Now consider the smooth map $\Phi^{\prime}: S^{n-1} / \Gamma \times(0, t) \rightarrow N-\{p\}$ defined by 
$\Phi(\theta, s)=\phi_{s}(\theta) \equiv \phi_{s}(\theta, 1)$. Then $\Phi^{\prime}$ may no longer be a diffeomorphism; however, this may easily be overcome as follows. Let $\rho$ be a smooth approximation to the distance function $r$ on $N-\{p\}$, such that $\left|\frac{\rho}{r}-1\right| \leq r^{2}$. Note that $\nabla \rho$ is transverse to all the "level hypersurfaces" $H_{s}=\operatorname{Im} \Phi^{\prime}(\cdot, s)$. Let $\psi_{t}(x)$ be the flow of $\nabla \rho$ and consider $\Phi: S^{n-1} / \Gamma \rightarrow N-\{p\}$ given by $\Phi(\theta, s)=\psi_{f(\theta, s)}\left(\phi_{s}(\theta)\right)$. Here $f(\theta, s)$ is uniquely determined by the requirement that $\Phi(\theta, s) \in L_{s} \equiv \rho^{-1}(s)$. It is then clear that $\Phi$ is a diffeomorphism onto its image. Note also that $|f(\theta, s)| \leq \varepsilon(s) \cdot s$. Now it is easily seen that $\Phi$ also satisfies (5.9), i.e.,

$$
\Phi^{*} g_{\infty}=\frac{(r \circ \Phi)^{2}}{r_{0}^{2}} \cdot g+o\left(r_{0}^{2}\right)
$$

We may lift the metrics $\Phi^{*} g_{\infty}$ and $g_{0}$ to the universal cover $B^{n}-\{0\}$ of $N$ and $C\left(S^{n-1} / \Gamma\right)-\{0\}$ and also lift $\Phi$ to a $\Gamma$-equivariant diffeomorphism $\Phi$ of $B^{n}-\{0\}$. Then (5.10) shows that the metric $\Phi^{*} g_{\infty}$ on $B^{n}-\{0\}$ has a $C^{0}$ extension over $\{0\}$. It can actually be arranged that $\Phi^{*} g_{\infty}$ has a $C^{1}$ extension over $\{0\}$, but we will not do this here.

It follows that $\Phi^{*} g_{\infty}$, which we will just call $g_{\infty}$, is a weak $\left(C^{0}\right)$ solution to the Einstein equation (4.1). At this stage, the regularity theory is not sufficient to imply that $g_{\infty}$ is smooth. However, we have $\int_{N}|R|^{n / 2}<\infty$, where $R$ is the curvature tensor of $g_{\infty}$. This situation has been treated by a number of authors, and we basically refer to these. For example, if $n \geq 5$, an elementary method of Sibner [34, Lemma 2.1, Proposition 2.4], which requires only a bound on the Sobolev constant on $N-p$, may easily be seen to apply to the present setting and shows that $R \in L^{p}$ for some $p>n / 2$. If $n=4$, using (5.5) and the Sobolev bound in $N-p$, one may verify step-by-step that the basic methods of Uhlenbeck [36, Theorem 4.1] remain valid here also, and show $R \in L^{p}$ for some $p>2$. It now follows from standard elliptic theory [28, Chapter $6 ; 36$, Theorem 3.6] that $g_{\infty}$ does extend smoothly across 0 . For more details in the proof of this, we refer, for instance, to [2]. This completes the main part of the proof of Theorem C.

We note that, even if $M_{\infty}$ is a smooth manifold with smooth Einstein metric, it does not follow from the proof above that $M_{i} \rightarrow M_{\infty}$ smoothly. In fact, arbitrarily small neighborhoods of the points $x_{k}(i) \in B_{k}^{i} \subset M_{i}$, with $x_{k}(i)$ converging to a curvature singularity $p_{k} \in M_{\infty}$, will have nontrivial topology (as we shall see later). This topology is "squeezed" or "bubbled" off as $i \rightarrow \infty$, regardless of whether $M_{\infty}$ is a smooth manifold or not.

For each curvature singularity $p \in\left\{p_{k}\right\} \subset M_{\infty}$, there is a sequence $x_{i} \in M_{i}$ such that $x_{i} \rightarrow p$ and $\inf _{r>0} \sup \left\{\left|K_{i}(x)\right|: x \in B_{x_{i}}(r) \subset M_{i}\right\} \rightarrow \infty$, as $i \rightarrow \infty$. Since the curvature of $M_{i}$ remains bounded in bounded distances away from $x_{i}$, we may assume that $x_{i}$ realizes the maximum $R_{i}$ of $|K(x)|$ on $B_{x_{i}}\left(r_{0}\right)$ for $r_{0}$ small. Now consider the pointed connected Riemannian manifolds 
$V_{i}=\left(B_{x_{i}}\left(r_{0}\right), x_{i}, R_{i}^{1 / 2} d s_{M_{i}}^{2}\right)$. We note that the curvature of $V_{i}$ is uniformly bounded and $\left|K\left(x_{i}\right)\right|=1$. Similarly, $\int_{V_{i}}|R|^{n / 2} \leq C$ and the Sobolev constants for $\left\{V_{i}\right\}$ are uniformly bounded below, since this is true for $M_{i}$ itself. As in the proof of Theorems $\mathrm{A}$ and $\mathrm{A}^{\prime}$, it follows that a subsequence of $\left\{V_{i}\right\}$ converges, smoothly in the $C^{k}$ topology on compact sets, to a complete connected Riemannian manifold $V\left(=V_{p}\right)$ satisfying

(i) $\quad \operatorname{Ric}_{V}=0$,

$$
\begin{aligned}
& \text { (ii) } \operatorname{vol} B(r) / r^{n} \geq C, \\
& \text { (iii) } \int_{V}|R|^{n / 2} \leq K, \\
& \text { (iv) }\left|K\left(x_{0}\right)\right|=1 \text {, for some } x_{0} \in V .
\end{aligned}
$$

Definition 5.1. A complete connected Riemannian manifold $V$ satisfying (5.11), obtained in the above manner, will be called an EALE (Einstein, asymptotically locally Euclidean) space associated to the curvature singularity $p \in$ $M_{\infty}$.

We note that, a priori, there may be more than one EALE space associated with $p \in M_{\infty}$. By construction, if $\Omega$ is a domain with smooth boundary in $V$, then there are smooth domains $\Omega_{i} \subset M_{i}$ and diffeomorphisms $\phi_{i}: \Omega \rightarrow \Omega_{i}$ such that $\phi_{i}^{*}\left(\left.r_{i} g_{i}\right|_{\Omega_{i}}\right)$ converges smoothly to $\left(\Omega, g_{V}\right)$, for an appropriate scaling $r_{i} \rightarrow \infty$. Note that $V$ cannot be diffeomorphic to a ball, by Theorem 3.5, so that $\Omega_{i}$ contains nontrivial topology squeezed off in the limit (cf. also Lemma 6.3 for the 4-dimensional case).

Now, to complete the proof of Theorem C, we recall from Theorem 3.5 that in odd dimensions, nontrivial, i.e., nonflat, EALE spaces do not exist, so that it follows that there are no curvature singularities in $M_{\infty}$. It follows that $M_{\infty}$ is a smooth manifold and the convergence $M_{i} \rightarrow M_{\infty}$ is smooth.

Remark. Some statements of Kobayashi and Todorov [27] indicate that Theorem $C$ is sharp in the sense that they exhibit examples of sequences of Einstein metrics satisfying the bounds (i), (iii) (and apparently (ii)), which converge to an Einstein orbifold in the above sense. More precisely, let $T^{4}=\mathbf{C}^{2} / \mathbf{Z}^{4}$, where $\mathbf{Z}^{4}$ is the standard (square) lattice in $\mathbf{C}^{2}$. The antipodal map $A: \mathbf{C}^{2} \rightarrow \mathbf{C}^{2}$ preserves the lattice and the quotient $X=T^{4} / \mathbf{Z}_{2}$ defines a flat orbifold, with flat singular metric $g_{X}$ having sixteen singular points, each a cone on $\mathbf{R P}^{3}$. If these sixteen points are blown up, one obtains a smooth 4-manifold $Y$ which is a $\mathrm{K} 3$ surface. Kobayashi and Todorov [27] then indicate that there are sequences of Ricci-flat Kahler metrics $g_{i}$ on $Y$ such that $g_{i} \rightarrow g_{X}$ in the Hausdorff distance, with convergence $g_{i} \rightarrow g_{\infty}$ smooth away from the singular points.

Finally, we note that by (5.1), the number of singular points of $M_{\infty}$ is bounded above by the bounds (i)-(iii) of Theorem C. Similarly, the order $\left|\Gamma_{j}\right|$ of each group $\Gamma_{j}$ associated with a singular point $p_{j}$ is bounded above. Namely, note that $g_{\infty}$ lifts to an Einstein metric on $B^{n} \backslash\{0\}$, which extends, 
modulo $\operatorname{Diff}\left(B^{n}-\{0\}\right)$, to a smooth metric on $B^{n}$. Thus, $\operatorname{vol}\left(B_{0}(r), g_{\infty}\right) \leq$ $c_{1} \cdot r^{n}$, where $B_{0}(r)$ is the geodesic ball of radius $r$ about 0 in the $g_{\infty}$ metric. Here we assume $r \leq r_{0}$, so that $c_{1}=\sinh ^{n-1} r_{0}$. Thus, if $B_{p}(r)$ is the $r$-ball about $p \in M_{\infty}$, then vol $B_{p}(r) \leq c_{1} r^{n} /|\Gamma|$. However, by the bounds (i), (ii) in Theorem $\mathrm{C}$, there is a uniform lower bound for the Sobolev constant and thus for the volume of small geodesic balls in $M_{i}$. It follows that vol $B_{x}(r / 4) \geq c_{2} r^{n}$ for $x \in S_{p}\left(\frac{r}{2}\right)$. This shows that $|\Gamma|$ is bounded by $c_{1} \cdot c_{2}$.

\section{COMPACTNESS OF MODULI SPACES}

In this section, we consider the question of the compactness, in a smooth topology, of the moduli space of positive Einstein metrics on compact 4-manifolds. Basically, the relation

$$
\chi(M)=\frac{1}{8 \pi^{2}} \int_{M}|R|^{2}
$$

for Einstein metrics in dimension 4 leads to further consequences of Theorem C.

Proposition 6.1. Let $\left(M_{i}, g_{i}\right)$ be a sequence of 4-dimensional Einstein manifolds satsfying the conditions of Theorem $\mathrm{C}$. Then

$$
\underline{\lim } \chi\left(M_{i}\right)=\underline{\lim } \frac{1}{8 \pi^{2}} \int_{M_{i}}|R|^{2} \geq \frac{1}{8 \pi^{2}} \int_{M_{\infty}}|R|^{2}+\sum_{1}^{Q} \int_{V_{j}}|R|^{2},
$$

where $Q$ is the number of curvature singularities of $M_{\infty}(c f .(5.4))$ and $V_{j}$ are associated EALE spaces (cf. Definition 5.1). Further, if $\underline{\lim \chi} \chi\left(M_{i}\right)=$ $\frac{1}{8 \pi^{2}} \int_{M_{\infty}}|R|^{2}$, then $M_{\infty}$ is a smooth manifold diffeomorphic to $M_{i}$ for $i$ sufficiently large, and the convergence $M_{i} \rightarrow M_{\infty}$ is smooth.

Proof. By Theorem C, a subsequence of $\left(M_{i}, g_{i}\right)$, also called $\left(M_{i}, g_{i}\right)$, converges to an Einstein orbifold $M_{\infty}$. Let $G_{i}^{r}$ and $B_{i}^{r}$ be defined as in the proof of Theorem C, $M_{i}=G_{i}^{r} \cup B_{i}^{r}$. Then

$$
\lim _{i \rightarrow \infty} \int_{M_{i}}|R|^{2}=\lim _{i \rightarrow \infty} \int_{G_{i}^{r}}|R|^{2}+\lim _{i \rightarrow \infty} \int_{B_{i}^{r}}|R|^{2}=\int_{G^{r}}|R|^{2}+\lim _{i \rightarrow \infty} \int_{B_{i}^{r}}|R|^{2},
$$

since the convergence $G_{i}^{r} \rightarrow G^{r}$ is smooth. On the other hand, since $\int|R|^{2}$ is scale-invariant, $\lim _{i \rightarrow \infty} \int_{B_{i}^{r}}|R|^{2} \geq \int_{V_{i}}|R|^{2}$, where $V_{i}$ is an EALE space associated with $p_{i}$. Since $r$ is arbitrary, this proves the first statement. For the second statement, the equality implies there are no nontrivial EALE spaces associated to $M_{\infty}$, and thus no curvature singularities of $M_{\infty}$. This implies by the proof of Theorem C that $M_{i} \rightarrow M_{\infty}$ smoothly.

Since the metric on $M_{\infty}$ extends (smoothly in the local universal cover) over the singularities, $\left(1 / 8 \pi^{2}\right) \int_{M_{\infty}}|R|^{2}$ may be identified with the orbifold Euler characteristic of $M_{\infty}$. Namely, if we excise small balls $B_{p_{i}}(r) \subset M_{\infty}$ around 
the singular points and let $M_{r}=M_{\infty}-\bigcup B_{p_{i}}$, then the Gauss-Bonnet theorem for manifolds with boundary [17] gives

$$
\chi\left(M_{r}\right)=\frac{1}{8 \pi^{2}} \int_{M_{r}}|R|^{2}+\int_{\partial M_{r}} \omega,
$$

where $\omega$ is a 3-form on $\partial M_{r}$ depending on $R$ and the second fundamental form of $\partial M_{r}$. Since $|R|$ is bounded on $M_{\infty}, \int_{M_{r}}|R|^{2} \rightarrow \int_{M_{\infty}}|R|^{2}$ and $\chi\left(M_{r}\right)=\chi\left(M_{0}\right)$, where $M_{0}=M_{\infty}-\left\{p_{i}\right\}$. Using the fact that each ball $B_{p_{i}}(r)$ is covered by a ball in $\mathbf{R}^{n}$ with smooth metric, one calculates that

$$
\int_{\partial M_{r}} \omega \rightarrow-\sum_{1}^{Q} \frac{1}{\left|\Gamma_{j}\right|}
$$

as $r \rightarrow 0$, where $\Gamma_{j}$ is the fundamental group of $B_{p_{j}}(r)-p_{j}$. Thus,

$$
\frac{1}{8 \pi^{2}} \int_{M_{\infty}}|R|^{2}=\chi\left(M_{0}\right)+\sum_{1}^{Q} \frac{1}{\left|\Gamma_{j}\right|}=\chi\left(M_{\infty}\right)
$$

where $\chi\left(M_{\infty}\right)$ is the orbifold Euler characteristic of $M_{\infty}$.

Similarly, $\int_{V}|R|^{2}$ also admits a topological interpretation. If $V_{r}$ denotes a geodesic ball of radius $r$ in $V$, then as before we have

$$
\chi\left(V_{r}\right)=\frac{1}{8 \pi^{2}} \int_{V_{r}}|R|^{2}+\int_{\partial V_{r}} \omega .
$$

For $r$ sufficiently large, $\chi\left(V_{r}\right)=\chi(V)$ and clearly, $\int_{V_{r}}|R|^{2} \rightarrow \int_{V}|R|^{2}$. Since $V$ is asymptotically flat, one may verify that

$$
\int_{\partial V_{r}} \omega \rightarrow \frac{1}{|\Theta|}
$$

where $|\Theta|$ is the order of the fundamental group of $V$ at infinity. Thus,

$$
\frac{1}{8 \pi^{2}} \int_{V}|R|^{2}=\chi(V)-\frac{1}{|\Theta|} \text {. }
$$

Recall from Theorem 3.5 that $|\Theta|=1$ if and only if $V$ is isometric to $\mathbf{R}^{4}$. Summarizing, we obtain

Corollary 6.2. Let $\left(M_{i}, g_{i}\right)$ be a sequence of Einstein metrics as above. Then

$$
\lim \chi\left(M_{i}\right) \geq \chi\left(M_{0}\right)+\sum_{1}^{Q} \frac{1}{\left|\Gamma_{j}\right|}+\sum_{1}^{Q}\left[\chi\left(V_{j}\right)-\frac{1}{\left|\Theta_{j}\right|}\right] .
$$

Lemma 6.3. If $V$ is a 4-dimensional EALE space, then $\chi(V) \geq 1$, with equality if and only if $V$ is isometric to $\mathbf{R}^{4}$.

Proof. Since $V$ is open, $\chi(V)=1-b_{1}+b_{2}-b_{3}$. Since $V$ is Ricci flat and asymptotically locally Euclidean, $\pi_{1}(V)$ is a finite group [1], so that $b_{1}=0$. By the exact homology sequence of the pair $(V, \partial V)=\left(V, S^{3} / \Gamma\right)$, one has

$$
0 \rightarrow H_{4}(V, \partial V) \rightarrow H_{3}(\partial V) \rightarrow H_{3}(V) \rightarrow H_{3}(V, \partial V) .
$$


By Poincare duality, it then follows that $H_{3}(V)=0$, so that $\chi(V)=1+$ $b_{2}$. Now suppose $b_{2}=0$. Then a finite cover $V^{\prime}$ of $V$ is a manifold with boundary, with all $\mathbf{Z}$-homology groups zero (since $V^{\prime}$ has no torsion in $H_{2}$ ). For convenience, we drop the prime and assume $V$ is an acyclic 4-manifold with boundary $\partial V=S^{3} / \Gamma, \Gamma \subset \mathrm{SO}(4)$. It is easily seen that $\partial V$ is a homology 3 -sphere, since, by the exact homology sequence, we have

$$
H_{1}\left(S^{3} / \Gamma ; \mathbf{Z}\right) \approx H_{2}\left(V, S^{3} / \Gamma ; \mathbf{Z}\right) \approx H^{2}(V ; \mathbf{Z})=0 .
$$

It is well known [39, p. $181 \mathrm{ff}$.] that the only spherical space forms with perfect $\pi_{1}$ are $S^{3}$ and the Poincare homology sphere $S^{3} / \Gamma_{0}$, where $\Gamma_{0}$ is the binary icosohedral group of order 120 .

There are two methods of proof to rule out the second case. First, by (6.3), we have

$$
\frac{1}{8 \pi^{2}} \int_{V}|R|^{2}=\chi(V)-\frac{1}{\left|\Gamma_{0}\right|}=1-\frac{1}{\left|\Gamma_{0}\right|} .
$$

On the other hand, one has a comparable expression for the signature $\tau$ of $V$, cf., e.g., [15],

$$
0=\tau(V)=\frac{1}{12 \pi^{2}} \int_{V}\left|R^{+}\right|^{2}-\left|R^{-}\right|^{2}+\left(-1+\frac{1}{\left|\Gamma_{0}\right|}\right)+\eta\left(S^{3} / \Gamma_{0}\right),
$$

where $\eta\left(S^{3} / \Gamma_{0}\right)$ is the eta invariant and $R^{+}\left(R^{-}\right)$are the self-dual (antiselfdual) components of $R$. One computes [15] that $\eta\left(S^{3} / \Gamma_{0}\right)=1079 / 360$. Thus,

$$
\begin{aligned}
1-\frac{1}{\left|\Gamma_{0}\right|}-\frac{1079}{360} & =\frac{1}{12 \pi^{2}} \int_{V}\left|R^{+}\right|^{2}-\left|R^{-}\right|^{2} \\
& \geq-\frac{2}{3}\left(\frac{1}{8 \pi^{2}} \int_{V}|R|^{2}\right)=-\frac{2}{3}\left(1-\frac{1}{\left|\Gamma_{0}\right|}\right),
\end{aligned}
$$

i.e.,

$$
\frac{5}{3}\left(1-\frac{1}{\left|\Gamma_{0}\right|}\right) \geq \frac{1079}{360} .
$$

This is clearly impossible, so that $S^{3} / \Gamma_{0}$ cannot bound an acyclic EALE space.

A second proof, purely topological, follows from work of Freedman and Donaldson. Namely, the Poincare homology 3-sphere cannot bound a smooth contractible 4-manifold, since it bounds a smooth 4-manifold $W$ with definite intersection form, equal to the $E_{8}$ lattice. In fact, $W$ is obtained by plumbing the disc bundles over $S^{2}$ with $c_{1}=-2$ according to the Dynkin diagram of $E_{8}$. (The interested reader may refer to [18, Problem section] to pursue this.)

It follows that $\partial V=S^{3}$ and the result follows from Theorem 3.5.

Remark. Lemma 6.3 allows one to replace the hypothesis $l_{M} \geq l>0$ by $\mathscr{A}_{M} \geq$ $\mathscr{A}>0$ in Theorem A. Namely, proceeding as before in the proof of Theorem A, one obtains a nontrivial EALE space $N$. One then needs to show that the hypothesis $\mathscr{A}_{M} \geq \mathscr{A}>0$ leads to a contradiction. Now, by Lemma 6.3, $b_{2}(N) \neq 0$, so that there is an integral 2-cycle $\Sigma \subset N$, with $[\Sigma] \neq 0$ in $H_{2}(N ; \mathbf{R})$ 
and area $\Sigma=A>0$. Since $N$ is a smooth limit of rescaled metrics on $M_{i}$, it follows that there is a sequence of domains $D_{i}$ diffeomorphic to $N$ in $M_{i}$ and integral 2-cycles $\left[\Sigma_{i}\right] \neq 0$ in $H_{2}\left(D_{i}, \mathbf{R}\right)$, but area $\Sigma_{i} \rightarrow 0$, in $\left(M_{i}, g_{i}\right)$. Writing the Mayer-Vietoris sequence for the pair $\left(D_{i}, M_{i}-D_{i}\right)$, noting that $\partial D_{i} \approx S^{n-1} / \Gamma$, one sees

$$
H_{2}\left(D_{i} ; \mathbf{R}\right) \oplus H_{2}\left(M_{i}-D_{i} ; \mathbf{R}\right) \simeq H_{2}\left(M_{i} ; \mathbf{R}\right),
$$

so that $\left[\Sigma_{i}\right]$ is a nonzero integral class in $H_{2}\left(M_{i} ; \mathbf{R}\right)$. This gives the required contradiction.

Returning to the discussion at hand, it follows from Lemma 6.3 that if $V$ is a nontrivial EALE space then

$$
\chi(V)-1 /|\Theta| \geq 3 / 2 .
$$

The Eguchi-Hanson metric on $T S^{2}$ [13] provides an example where equality is achieved.

Theorem 6.4. Let $M$ be a compact 4-manifold of Euler characteristic $0<$ $\chi(M) \leq 3$ (e.g., $M=S^{4}$ or $\left.C P^{2}\right)$. Then the space of +1 Einstein metrics $g$ on $M$ such that $\operatorname{vol}_{g}(M) \geq c>0$ is compact in the $C^{k}$ topology. In particular, the components of positive Einstein metrics in the moduli space of Einstein metrics on $M$ are compact.

Proof. Let $\left\{g_{i}\right\}$ be a sequence of +1 Einstein metrics on $M$ such that $\operatorname{vol}_{g_{i}}(M)$ $\geq c$. It follows from Theorem $C$ that a subsequence, also called $\left\{g_{i}\right\}$, converges to an Einstein orbifold $\left(M_{\infty}, g_{\infty}\right)$. If the convergence $\left(M, g_{i}\right) \rightarrow\left(M_{\infty}, g_{\infty}\right)$ is not smooth, then by the proof of Theorem C, there are nontrivial EALE spaces $V$ associated to the curvature singularities of $M_{\infty}$. By (6.4) and (6.5), one obtains

$$
3 \geq \chi(M) \geq \chi\left(M_{0}\right)+\Sigma \frac{1}{|\Gamma|}+Q \cdot \frac{3}{2},
$$

where $M_{0}$ is $M_{\infty}$ with the curvature singularities removed and $Q$ is the number of singularities. Since

$$
\chi\left(M_{0}\right)+\Sigma \frac{1}{|\Gamma|}=\frac{1}{8 \pi^{2}} \int_{M_{\infty}}|R|^{2} \geq 0,
$$

we see that $Q \leq 2$. If $Q=2$, then for instance $\int_{M_{\infty}}|R|^{2}=0$, so that $M_{0}$ is flat. This is impossible, since the metric on $M_{0}$ is a +1 Einstein metric. If $Q=1$, then

$$
\chi\left(M_{0}\right)+1 /|\Gamma| \leq 3 / 2,
$$

so that $\chi\left(M_{0}\right) \leq 1$. Note that by Theorem C, $M_{0}$ is embedded as an open set in $M$. Further, $M_{0}$ has a boundary of the form $S^{3} / \Gamma$. Now since $M$ carries a metric of positive Ricci curvature, $\left|\pi_{1}(M)\right|<\infty$ and the Seifert-Van Kampen theorem, applied to $\left(M_{0}, M-M_{0}\right)$, shows that $\left|\pi_{1}\left(M_{0}\right)\right|<\infty$ also. In particular, $b_{1}\left(M_{0}\right)=0$, so that $\chi\left(M_{0}\right)=1$. The homology sequence arguments 
above show that there is a finite cover $M_{0}^{\prime}$ of $M_{0}$ with acyclic $\mathbf{Z}$-homology and $\partial M_{0}^{\prime}=S^{3}$. Since $\chi\left(M_{0}^{\prime}\right)=1$ also, and the Euler characteristic is multiplicative under finite covers, we see $M_{0}=M_{0}^{\prime}$. Thus, $\chi\left(M_{0}\right)=1, \partial M_{0}=S^{3}$, which contradicts $(6.6)$.

Thus, $Q=0$, so that there is no nontrivial EALE space associated to $M_{\infty}$. The result now follows from Proposition 6.1.

Remark. The method above does not work for $\chi(M)=4$. For instance, let $M=S^{2} \times S^{2}=T S^{2} \cup_{\partial T S^{2}}-T S^{2}$, where $T S^{2}$ is the tangent bundle to $S^{2}$. Then $\chi(M)=4$ and $M$ admits +1 Einstein metrics. We are not able to rule out the existence of a sequence of +1 Einstein metrics converging to an orbifold with one singular point (corresponding to a collapse of $T S^{2}$ to $C\left(\mathbf{R P}^{3}\right)$ ).

Finally, we mention one further result along these lines.

Theorem 6.5. Let $M$ be a compact 4-manifold. Then the space of +3 Einstein metrics on $M$ with volume $\operatorname{vol}_{M} \geq((1+\varepsilon) / 2) \cdot \operatorname{vol} S^{4}(1)$, for a fixed $\varepsilon>0$, is compact in the $C^{k}$ topology.

Proof. Let $g_{i}$ be a sequence of +3 Einstein metrics on $M$ such that $\operatorname{vol}_{g_{i}}(M)$ $\geq(1+\varepsilon) / 2$. By Theorem $\mathrm{C}$, a subsequence converges to an Einstein orbifold $M_{\infty}$. As above, if the convergence $\left(M, g_{i}\right) \rightarrow\left(M_{\infty}, g_{\infty}\right)$ is not smooth, then there is at least one nontrivial EALE space $V$ associated with $\left\{g_{i}\right\}$. If $v(r)$ is the volume of a geodesic $r$-ball $B(r)$ about $p_{0} \in V$, then $\lim _{r \rightarrow \infty} v(r) / r^{4}=$ vol $C\left(S^{3}(1) / \Gamma\right) \leq \frac{1}{2}$ vol $B^{4}(1)$, since $|\Gamma| \geq 2$. Given $\delta>0$, choose $R$ such that $v(r) / r^{4} \leq(1+\delta) / 2 \cdot \operatorname{vol} B^{4}(1), \forall r \geq R$. Now by construction, $B(r) \subset V$ is embedded in $M$, with metric a scaled limit of $\left\{g_{i}\right\}$. Since the ratio $v(r) / r^{4}$ is scale invariant, there are geodesic balls $B_{i}=B_{x_{i}}\left(\varepsilon_{i}\right) \subset\left(M, g_{i}\right)$, with $\varepsilon_{i} \rightarrow 0$, such that

$$
\frac{\operatorname{vol} B_{x_{i}}\left(\varepsilon_{i}\right)}{\varepsilon_{i}^{4}} \leq \frac{1+\delta}{2} \operatorname{vol} B^{4}(1)
$$

Since the Ricci curvature of $\left(M, g_{i}\right)$ is +3 , the Bishop comparison theorem implies that $\operatorname{vol} B_{x}(r) / v^{+3}(r)$ is monotone nonincreasing, where $v^{+3}(r)$ is the volume of a geodesic $r$-ball in $S^{4}(1)$. Now, by Myer's theorem, $\operatorname{diam}_{g_{i}}(M) \leq$ $\operatorname{diam}\left(S^{4}(1)\right)=\pi$, so we obtain

$$
\frac{\operatorname{vol} M}{\operatorname{vol} S^{4}(1)}=\frac{\operatorname{vol} B_{x_{i}}(\pi)}{\operatorname{vol} S^{4}(1)} \leq \frac{\operatorname{vol} B_{x_{i}}\left(\varepsilon_{i}\right)}{v^{+3}\left(\varepsilon_{i}\right)} \leq \frac{1+\delta}{2} \operatorname{vol} B^{4}(1) \cdot \frac{\varepsilon_{i}^{4}}{v^{+3}\left(\varepsilon_{i}\right)} .
$$

Clearly, $v^{+3}\left(\varepsilon_{i}\right) / \varepsilon_{i}^{4} \rightarrow \operatorname{vol} B^{4}(1)$ as $\varepsilon_{i} \rightarrow 0$. Since $\delta$ is arbitrary, this contradicts the hypothesis, and thus gives the result.

We remark that the major difficulty in extending the results above to Ricci-flat or negative Einstein metrics is obtaining control on the diameter of sequences of such metrics. 


\section{REFERENCES}

1. M. T. Anderson, On the topology of complete manifolds of non-negative Ricci curvature, Topology 28 (1989).

2. S. Bando, A. Kasue, and H. Nakajima, On a construction of coordinates at infinity on manifolds with fast curvature decay, Univ. of Tokyo, preprint.

3. J. Bemelmans, M. Min-Oo, and E. Ruh, Smoothing Riemannian metrics, Math. Z. 188 (1984), 69-74.

4. M. Berger, Sur les variétés Riemanniennes pincées juste du-dessous de $\frac{1}{4}$, Ann. Inst. Fourier (Grenoble) 33 (1983), 135-150.

5. A. Besse, Einstein manifolds, Ergeb. Math. Grenzgeb. Band 10, Springer-Verlag, Berlin and New York, 1987.

6. R. Bishop and R. Crittenden, Geometry of manifolds, Academic Press, New York, 1964.

7. E. Calabi, Métriques Kählériennes et fibrés holomophes, Ann. Sci. École Norm. Sup. (4) 12 (1979), 269-294.

8. J. Cheeger, Finiteness theorems for Riemannian manifolds, Amer. J. Math. 92 (1970), 61-74.

9. J. Cheeger, M. Gromov, and M. Taylor, Finite propagation speed, kernel estimates for functions of the Laplace operator and the geometry of complete Riemannian manifolds, J. Differential Geom. 17 (1982), 15-53.

10. C. Croke, Some isoperimetric inequalities and eigenvalue estimates, Ann. Sci. École Norm. Sup. (4) 13 (1980), 419-435.

11. D. Deturck and J. Kazdan, Some regularity theorems in Riemannian geometry, Ann. Sci. École Norm. Sup. (4) 14 (1980), 249-260.

12. D. Ebin, The manifold of Riemannian metrics, Proc. Sympos. Pure Math., Vol. 15, Amer. Math. Soc., Providence, RI, 1970, pp. 11-40.

13. T. Eguchi and A. Hanson, Asymptotically flat self-dual solutions to Euclidean gravity, Phys. Lett. B 74 (1978), 249-251.

14. L. Z. Gao, Einstein metrics, preprint.

15. G. Gibbons, C. Pope, and A. Romer, Index theorem boundary terms for gravitational instantons, Nuclear Phys. B 157 (1979), 377-386.

16. D. Gilbarg and N. Trudinger, Elliptic partial differential equations of second order, SpringerVerlag, New York, 1977.

17. P. Gilkey, The heat equation and the Atiyah-Singer index theorem, Publish or Perish, Wilmington, DE, 1986.

18. C. Gordon and R. Kirby, Four-manifold theory, Contemp. Math., Vol. 35, Amer. Math. Soc., Providence, RI, 1984.

19. R. Greene and H. Wu, Lipschitz convergence of Riemannian manifolds, Pacific J. Math. 131 (1988), 119-141.

20. M. Gromov, Structures métriques pour les variétés Riemanniennes, Cedic/Fernand Nathan, Paris, 1981.

21. R. Hamilton, Three-manifolds with positive Ricci curvature, J. Differential Geom. 17 (1982), 255-306.

22. N. Hitchin, Polygons and gravitons, Math. Proc. Cambridge Philos. Soc. 85 (1979), 465-476.

23. J. Jost and H. Karcher, Geometrische Methoden fur gewinnung von a-priori schranken fur harmonische abbildungen, Manuscripta Math. 40 (1982), 27-71.

24. A. Kasue, A convergence theorem for Riemannian manifolds and some applications, Nagoya Math. J. 114 (1989).

25. A. Katsuda, Gromov's compactness theorem and its application, Nagoya Math. J. 100 (1985), 11-48.

26. W. Klingenberg, Contributions to Riemannian geometry in the large, Ann. of Math. (2) 69 (1959), 654-666. 
27. R. Kobayashi and A. Todorov, Polarized period map for generalized K3 and the moduli of Einstein metrics, Tohoku Math. J. 39 (1987), 341-363.

28. C. B. Morrey, Jr., Multiple integrals in the calculus of variations, Springer-Verlag, New York, 1966.

29. H. Nakajima, Hausdorff convergence of Einstein metrics on 4-manifolds, preprint.

30. P. Pansu, Effondrement des varietes riemanniennes d'apres J. Cheeger et M. Gromov, Astérisque 121 (1985).

31. S. Peters, Cheeger's finiteness theorem for diffeomorphism classes of Riemannian manifolds, $\mathbf{J}$. Reine Angew. Math. 349 (1984), 77-82.

32. J. Sachs and K. Uhlenbeck, The existence of minimal immersions of 2-spheres, Ann. of Math. (2) 113 (1981), 1-24.

33. R. Schoen, Analytic aspects of the harmonic map problem, Math. Sci. Res. Inst. Series, Vol. 2, Springer-Verlag, Berlin and New York, 1984.

34. L. Sibner, The isolated point singularity problem for the coupled Yang-Mills equation in higher dimensions, Math. Ann. 271 (1985), 125-131.

35. G. Tian and S.-T. Yau, Kähler-Einstein metrics on complex surfaces with $c_{1}>0$, Comm. Math. Phys. 112 (1987), 175-203.

36. K. Uhlenbeck, Removable singularities in Yang-Mills fields, Comm. Math. Phys. 83 (1982), 11-29.

37. _ Connections with $L^{p}$ bounds on curvature, Comm. Math. Phys. 83 (1982), 31-42.

38. M. Wang and W. Ziller, Einstein metrics with positive scalar curvature, Lecture Notes in Math., No. 1201, Springer-Verlag, New York, 1986.

39. J. Wolf, Spaces of constant curvature, Publish or Perish, Boston, MA, 1974.

40. S.-T. Yau, Survey lecture, Seminar on Differential Geom., Ann. of Math. Stud., Vol. 102, 1982.

Department of Mathematics, State University of New York, Stony Brook, New York 11794-3651

e-mail address: manderson @ sbccmail.bitnet 